\title{
A STABILITY THEOREM FOR PROJECTIVE CR MANIFOLDS
}

\author{
JUDITH BRINKSCHULTE, C. DENSON HILL, AND MAURO NACINOVICH
}

\begin{abstract}
We consider smooth deformations of the $C R$ structure of a smooth 2-pseudoconcave compact $C R$ submanifold $\mathrm{M}$ of a reduced complex analytic variety $X$ outside the intersection $D \cap M$ with the support $D$ of a Cartier divisor of a positive line bundle $F_{X}$. We show that nearby structures still admit projective $C R$ embeddings. Special results are obtained under the additional assumptions that $\mathrm{X}$ is a projective space or a Fano variety.
\end{abstract}

\section{Contents}

1. Introduction

2. Preliminaries and notation

2.1. $C R$ manifolds of type $(n, k)$

2.2. Natural projection onto the canonical bundle

2.3. $\quad C R$ maps

2.4. q-pseudoconcavity

2.5. Deformation of $C R$ structures

3. $\bar{\partial}_{\mathrm{M}}$-cohomology

3.1. Differential presentation of the tangential $C R$ complex

3.2. $C R$ line bundles

3.3. Deformations and $C R$ line bundles

4. An example

5. Vanishing results

5.1. Embedded $C R$ manifolds

5.2. Some estimates for abstract $C R$ manifolds

5.3. Deformation of tangential $C R$ complexes

5.4. Estimates for deformations

5.5. Vanishing theorems

6. Proof of Theorem 1.1

6.1. The general case

6.2. Generic $C R$ submanifolds of the projective space

6.3. Generic $C R$ submanifolds of Fano varieties

References 


\section{INTRODUCTION}

In this paper we study projective $C R$ manifolds, meaning those that have a $C R$ embedding into some $\mathbb{C} \mathbb{P}^{N}$. We consider a small $C R$ deformation of such an $\mathrm{M}$, and investigate under what conditions the deformed $C R$ manifold still has a projective $C R$ embedding. Our main result is the following $C R$ embedding theorem.

Theorem 1.1. Let $X$ be an $(n+k)$-dimensional reduced complex analytic variety and $D$ the support of a Cartier divisor of a positive line bundle $F_{X}$.

Let $M$ be a smooth compact submanifold of real dimension $2 n+k$ of $X_{\text {reg }}$ and $\left\{M_{t}\right\}_{t \in \mathbb{R}}$ a family of CR structures of type $(n, k)$ on $M$, smoothly depending on a real parameter $t$. We assume that

(1) $M_{0}$ is induced by the embedding $M \hookrightarrow X$;

(2) $M_{0}$ is 2-pseudoconcave;

(3) the CR structures of all $M_{t}$ agree to infinite order on $M \cap D$.

Then we can find $\epsilon_{0}>0$ such that, for every $|t|<\epsilon_{0}, M_{t}$ admits a generic $C R$ embedding into a projective complex variety $X_{t}$.

More precise results are obtained under the additional assumptions that $\mathrm{X}$ is a projective space or a Fano variety (Theorems 6.1 and 6.2).

The example of $\$ 4$ shows that an assumption of 1-pseudoconcavity on $M_{0}$ is not sufficient to obtain the statement of Theorem 1.1.

Precise definitions and explanation of terms used will be found in the following sections; let us start by explaining how our main theorem relates to other results in the field of CR geometry or analysis on CR manifolds.

The notion of a $C R$ structure of type $(n, k)$ on a differentiable manifold $\mathrm{M}$ of real dimension $2 n+k$ arises very naturally in two, a priori different, contexts. One is quite geometric in nature, and the other is more analytical, being connected with fundamental questions about partial differential equations.

From the geometric perspective, a $C R$ structure of type $(n, k)$ on a smooth real submanifold $\mathrm{M}$ of dimension $2 n+k$ of some complex manifold $\mathrm{X}$ of complex dimension $n+k$ is induced as the tangential part of the ambient complex structure on X. In particular, one has the tangential Cauchy-Riemann equations on $\mathrm{M}$. In this situation $\mathrm{M}$ is said to be generically $C R$ embedded in $\mathrm{X}$. It is also possible that $\mathrm{M}$ might be embedded in a complex manifold $X$ of complex dimension greater than $n+k$, with the $C R$ structure on $\mathrm{M}$ also being induced as the tangential part of the ambient complex structure in $\mathrm{X}$. In this situation the real codimension of $\mathrm{M}$ is greater than the $C R$ dimension $k$, and $\mathrm{M}$ is said to be non generically $C R$ embedded in $\mathrm{X}$. This is an important distinction only globally, because: if $\mathrm{M}$ can be locally non generically $\mathrm{CR}$ embedded, then M can be locally generically $C R$ embedded (see e.g. [25]). 
From the PDE perspective, one begins with (at least locally) a system of $\mathrm{n}$ smooth complex vector fields defined on $\mathrm{M}$, which are linearly independent over $\mathbb{C}$, and satisfy a formal integrability condition. Again $n$ is the $C R$ dimension, and $k$ is the $C R$ codimension. One then asks if the given complex vector fields might represent (in a local basis) the tangential $C R$ equations on $\mathrm{M}$ given by some (local) $C R$ embedding of $\mathrm{M}$. The criterion for this, is that one must be able to find (locally) a maximal set of functionally independent characteristic coordinates, which means to find $n+k$ independent complex valued functions that are solutions of the homogeneous system associated to the $n$ complex vector fields. When the vector fields can be chosen to have real analytic coefficients, then this is possible ([1]). Hence locally, in the real analytic case, the geometric perspective and the analytical PDE perspective are equivalent. The famous example of $\mathrm{L}$. Nirenberg [33] showed that the two perspectives are not locally equivalent if the coefficients of the vector fields are assumed to be only smooth. Why does this matter? From an analytical PDE viewpoint, it is too restrictive to allow only real analytic structures, one needs cut off functions, partitions of unity; etc., in order to employ calculus and modern analysis. So from this perspective it is more natural to define what is known as an abstract $C R$ manifold $\mathrm{M}$ of type $(n, k)$, by reformulating the local vector field basis description given above into a global and invariant version, and to do so within the smooth category. Precise definitions and details are below.

The example of Nirenberg [33] was a single complex vector field in $\mathbb{R}^{3}$, so it endows $\mathbb{R}^{3}$ with the structure of an abstract $C R$ manifold of type $(1,1)$. To make matters worse, the complex vector field of Nirenberg's example is a small smooth perturbation, near some point, of the tangential Cauchy Riemann equation to the unit sphere in $\mathbb{C}^{2}$. In other words, the generically $C R$ embedded and real analytic $C R$ structure of type $(1,1)$ on the sphere $S^{3}$, inherited from $\mathbb{C}^{2}$, becomes not even locally $C R$ embeddable near some point, if it is perturbed in a smooth way to obtain a very nearby abstract $C R$ structure of type $(1,1)$ on the same $S^{3}$. Here the original $C R$ structure on $S^{3}$ is strictly pseudoconvex, and remains so under the small perturbation.

Rossi [36] constructed small real analytic deformations of the $C R$ structure on $S^{3}$ in $\mathbb{C}^{2}$, such that the resulting perturbed abstract $C R$ structures fail to $C R$ embed globally into $\mathbb{C}^{2}$, even though they do locally $C R$ embed into $\mathbb{C}^{2}$. On the other hand, sticking with strictly pseudoconvex $C R$ structures of hypersurface type, i.e. type $(n, 1)$, Boutet de Monvel proved that when $\mathrm{M}$ is compact and $n>1$, then $\mathrm{M}$ has a $C R$ embedding into $\mathbb{C}^{N}$ for some perhaps large $N$. Catlin and Lempert [15] showed that there are compact strictly pseudoconvex $C R$ manifolds of hypersurface type $(n, 1)$, for $n \geq 1$, that are $C R$ embedded into some $\mathbb{C}^{N}$, which admit small deformations that are also embeddable, but their embeddings cannot be chosen close to the original embedding. Thus these examples exhibit the phenomenon of instability of the $C R$ embedding. On the other hand, for $n=1$, Lempert [31] proved that if a compact strictly pseudoconvex $\mathrm{M}$ is $C R$ embeddable 
into $\mathbb{C}^{2}$, hence generically embedded, and if the $C R$ structure gets perturbed by a family of $C R$ embeddable $C R$ structures, then the embedding is stable. This means that the embedding of the perturbed structure stays close to the unperturbed one. Additional references to other work on the strictly pseudoconvex hypersurface type case can be found in the above mentioned papers.

In the higher codimensional case $(k>1)$ Polyakov [35] proved a stability theorem for compact 3-pseudoconcave $C R$ manifolds $\mathrm{M}$, generically $C R$ embedded in a complex manifold, under some additional hypotheses, such as the vanishing of a certain cohomology group. The notion of flexible/inflexible, which is closely related to the notion of stability/instability, was introduced in [10], also for the higher codimension case, where the emphasis was on $C R$ manifolds $\mathrm{M}$ that are not compact. A $C R$ manifold $\mathrm{M}$ of type $(n, k)$, generically $C R$ embedded in $\mathbb{C}^{n+k}$, is called flexible if it admits a compactly supported $C R$ deformation whose deformed structure is no longer $C R$ embeddable in $\mathbb{C}^{n+k}$; in other words, the deformation causes the $C R$ structure to "flex out" of the space where the original manifold lies. On the other hand, $\mathrm{M}$ is inflexible if any compactly supported deformation stays in the class of manifolds globally $C R$ embeddable into $\mathbb{C}^{n+k}$. In [10], [11] it was proved that if $\mathrm{M}$ is a $C R$ submanifold of type $(n, k)$ in $\mathbb{C}^{n+k}$, and it is only 2-pseudoconcave, then $\mathrm{M}$ is inflexible. This relates to the present paper as follows: If a $C R$ structure of a 2-pseudoconcave compact $C R$ manifold of a reduced complex analytic variety gets perturbed by a smooth family of $C R$ structures that "glues to" the unperturbed structure along a Cartier divisor of a positive line bundle, then the perturbed $C R$ structures do not "flex out" of the class of globally $C R$ embeddable $C R$ manifolds (Theorem 1.1). Moreover, the embedding is stable if the unperturbed $C R$ manifold sits generically in a projective space (Theorem6.1).

We emphasize that in this paper we discuss compact $C R$ manifolds and we allow higher codimension and that we are interested in global results, not local ones.

\section{Preliminaries AND NOtation}

2.1. $C R$ manifolds of type $(n, k)$. Let $\mathrm{M}$ be a smooth manifold of real dimension $m$ and $n, k$ nonnegative integers with $2 n+k=m$. A $C R$ structure of type $(n, k)$ on $\mathrm{M}$ is a formally integrable distribution $\mathrm{T}_{\mathrm{M}}^{0,1}$ of $n$-dimensional complex subspaces of the complexified tangent bundle $\mathbb{C T}_{\mathrm{M}}$ of $\mathrm{M}$, transversal to $T_{M}$. By this transversality condition, the real parts of vectors in $T_{M}^{0,1}$ are the elements of the vector $C R$ distribution $\mathrm{H}_{\mathrm{M}}$ and for each $\mathrm{p} \in \mathrm{M}$ and $v \in \mathrm{H}_{\mathrm{M}, \mathrm{p}}$ (subscript "p" means "the fiber at p") there is a unique $v^{\prime} \in \mathrm{H}_{\mathrm{M}, \mathrm{p}}$ such that $v+i v^{\prime} \in \mathrm{T}_{\mathrm{M}, \mathrm{p}}^{0,1}$. The correspondence $v \rightarrow v^{\prime}$ defines a bundle self map $J_{M}: H_{M} \rightarrow H_{M}$ which yields a complex structure on the fibers of $H_{M}$, as $\mathrm{J}_{\mathrm{M}}^{2}=-\mathrm{I}_{\mathrm{H}_{\mathrm{M}}}$. The formal integrability condition means that

$$
\left[\Gamma^{\infty}\left(\mathrm{M}, \mathrm{T}_{\mathrm{M}}^{0,1}\right), \Gamma^{\infty}\left(\mathrm{M}, \mathrm{T}_{\mathrm{M}}^{0,1}\right)\right] \subseteq \Gamma^{\infty}\left(\mathrm{M}, \mathrm{T}_{\mathrm{M}}^{0,1}\right),
$$


which is equivalent to the vanishing of the Nijenhuis tensor on $\mathrm{H}_{M}$, i.e.

$$
\mathrm{J}_{\mathrm{M}}\left([X, Y]-\left[\mathrm{J}_{\mathrm{M}} X, \mathrm{~J}_{\mathrm{M}} Y\right]\right)=\left[\mathrm{J}_{\mathrm{M}} X, Y\right]+\left[X, \mathrm{~J}_{\mathrm{M}} Y\right], \quad \forall X, Y \in \Gamma^{\infty}\left(\mathrm{M}, \mathrm{H}_{\mathrm{M}}\right) .
$$

We use the standard notation

$$
\begin{aligned}
\mathrm{T}_{\mathrm{M}}^{1,0} & =\left\{X-i \mathrm{~J}_{\mathrm{M}} X \mid X \in \mathrm{H}_{\mathrm{M}}\right\} \subset \mathbb{C T}_{\mathrm{M}}, \\
\mathrm{T}_{\mathrm{M}}^{0,1} & =\left\{X+i \mathrm{~J}_{\mathrm{M}} X \mid X \in \mathrm{H}_{\mathrm{M}}\right\} \subset \mathbb{C T}_{\mathrm{M}}, \\
\mathrm{T}_{\mathrm{M}}^{* 1,0} & =\left\{\phi \in \mathbb{C T}_{\mathrm{M}}^{*} \mid \phi(Z)=0, \forall Z \in \Gamma^{\infty}\left(\mathrm{M}, \mathrm{T}_{\mathrm{M}}^{0,1}\right)\right\}, \\
\mathrm{T}_{\mathrm{M}}^{* 0,1} & =\left\{\phi \in \mathbb{C T}_{\mathrm{M}}^{*} \mid \phi(Z)=0, \forall Z \in \Gamma^{\infty}\left(\mathrm{M}, \mathrm{T}_{\mathrm{M}}^{1,0}\right)\right\}, \\
\mathrm{H}_{\mathrm{M}}^{0} & =\left\{\xi \in \mathrm{T}_{\mathrm{M}}^{*} \mid \xi(X)=0, \forall X \in \Gamma^{\infty}\left(\mathrm{M}, \mathrm{H}_{\mathrm{M}}\right)\right\} .
\end{aligned}
$$

We have

$$
\mathrm{T}_{\mathrm{M}}^{1,0} \cap \mathrm{T}_{\mathrm{M}}^{0,1}=\{0\}, \quad \mathrm{T}_{\mathrm{M}}^{1,0}+\mathrm{T}_{\mathrm{M}}^{0,1}=\mathbb{C H}_{\mathrm{M}}, \quad \mathrm{T}_{\mathrm{M}}^{* 1,0} \cap \mathrm{T}_{\mathrm{M}}^{* 0,1}=\mathbb{C H}_{\mathrm{M}}^{0}, \quad \mathrm{~T}_{\mathrm{M}}^{* 1,0}+\mathrm{T}_{\mathrm{M}}^{* 0,1}=\mathbb{C T}_{\mathrm{M}}^{*} .
$$

Deformations of $C R$ structures were considered especially for the hypersurface-type case $k=1$ [2, 17]. Since, by a theorem of Darboux (see [16]), 1 -codimensional contact structures are locally equivalent, deformations of non degenerate $C R$ hypersurfaces can be described by smooth curves $\left\{\mathrm{t} \rightarrow \mathrm{J}_{\mathrm{t}}\right\}$ of formally integrable complex structures on a fixed contact distribution $\mathrm{H}_{M}$ of a smooth real manifold M. Differently, generalized contact distributions of higher codimension may be not locally equivalent. Therefore, to describe general deformations of abstract $C R$ manifolds of type $(n, k)$ with arbitrary $k$ it will be convenient to focus our attention on their canonical bundles, which carry comprehensive information on both their contact and partial complex structures, as explained below.

Denote by $\mathcal{A}_{\mathrm{M}}$ the sheaf of Grassmann algebras of germs of complex valued smooth exterior differential forms on $\mathrm{M}$. The subsheaf $\mathcal{A}_{\mathrm{M}}^{q}$ of homogeneous elements of degree $q$ consists of the germs of smooth sections of the $\mathbb{C}$-vector bundle $\Lambda^{q}\left(\mathbb{C T}_{\mathrm{M}}^{*}\right)$ of complex $q$-covectors on $M$.

We can identify the Grassmannian $\mathcal{G}_{n}^{\mathbb{C}}(\mathrm{M})$ of $n$-dimensional complex subspaces of $\mathbb{C} \mathrm{T}_{\mathrm{M}}$ with the subbundle of the projective bundle $\mathbb{P}\left(\Lambda^{n+k}\left(\mathbb{C T}_{\mathrm{M}}^{*}\right)\right)$ whose representatives belong to the cone $\mathcal{M}$ on $\left(\Lambda^{n+k}\left(\mathbb{C T}^{*} M\right)\right)$ of degree $n+k$ monomials] of the Grassmann algebra of $\mathbb{C T}^{*} M$ (see e.g. [14]). Then a distribution of complex $n$-planes of $\mathbb{C} \mathrm{T}_{\mathrm{M}}$ is the datum of a smooth complex line bundle $\mathrm{K}_{\mathrm{M}}$ on $\mathrm{M}$, with $\mathrm{K}_{\mathrm{M}} \subset \mathcal{M}$ on $\left(\Lambda^{n+k}\left(\mathbb{C T}^{*} M\right)\right)$.

Definition 2.1. Let $\left(M, \mathrm{~T}_{\mathrm{M}}^{0,1}\right)$ be an abstract $C R$ manifold of type $(n, k)$. The complex line bundle $\mathrm{K}_{\mathrm{M}}$ corresponding to the distribution $\mathrm{T}_{\mathrm{M}}^{0,1}$ of its antiholomorphic tangent vectors is called its canonical bundle.

Notation 2.1. Let us denote by $\mathcal{L}_{M}$ the subsheaf of ideals in $\mathcal{A}_{M}$ generated by the sheaf $\underline{K}_{M}$ of germs of smooth sections of $K_{M}$.

\footnotetext{
${ }^{1} \mathrm{We}$ recall that, if $\mathrm{V}$ is a vector space, the monomials of degree $q$ of its exterior algebra $\Lambda^{*} \mathrm{~V}$ are the exterior products $v_{1} \wedge \cdots \wedge v_{q}$ of vectors $v_{1}, \ldots, v_{q}$ of $\mathrm{V}$.
} 
Proposition 2.1. A CR structure of type $(n, k)$ on $M$ is completely determined by the datum of a complex line bundle $K_{M} \subset \mathcal{M}$ on $\left(\Lambda^{n+k}\left(\mathbb{C T}_{M}^{*}\right)\right)$ such that the ideal sheaf $\mathcal{L}_{M}$ of $\mathcal{A}_{M}$ generated by the germs its smooth sections satisfies the following conditions:

(1) $\mathcal{K}_{M}$ is closed, i.e. $d \mathcal{K}_{M} \subseteq \mathcal{K}_{M} \quad$ (FORMAL INTEGRABILITY);

(2) $\left.\left\{X \in \Gamma^{\infty}\left(M, \mathrm{~T}_{M}\right) \mid X\right\rfloor \mathcal{K}_{M} \subseteq \mathcal{K}_{M}\right\}=\{0\} \quad$ (transversality to $\mathrm{T}_{\mathrm{M}}$ ).

Indeed, when (1) and (2) are valid, $K_{M}$ is the canonical bundle of the $C R$ manifold $\left(M, \mathrm{~T}_{M}^{0,1}\right)$ with

$$
\left.\Gamma^{\infty}\left(M, \mathrm{~T}_{M}^{0,1}\right)=\left\{Z \in \Gamma^{\infty}\left(M, \mathbb{C T}_{M}\right) \mid Z\right\rfloor \mathcal{K}_{M} \subseteq \mathcal{K}_{M}\right\}
$$

Proof. In fact, (1) is equivalent to the formal integrability (2.1) and (2) to the transversality of $\mathrm{T}_{\mathrm{M}}^{0,1}$ to the real tangent distribution $\mathrm{T}_{\mathrm{M}}$.

By the Newlander-Nirenberg theorem ([34]) abstract $C R$ manifolds of type $(n, 0)$ are $n$-dimensional complex manifolds.

2.2. Natural projection onto the canonical bundle. On a $C R$ manifold $M$ of type $(n, k)$ we have an exact sequence of smooth bundle maps

$$
0 \longrightarrow \mathbb{C H}_{\mathrm{M}}^{0} \longrightarrow \mathrm{T}_{\mathrm{M}}^{* 1,0} \oplus \mathrm{T}_{\mathrm{M}}^{* 0,1} \longrightarrow \mathbb{C T}_{\mathrm{M}}^{*} \longrightarrow 0,
$$

where

$$
\begin{gathered}
\mathbb{C H}_{\mathrm{M}}^{0} \ni \xi \longrightarrow(\xi, \xi) \in \mathrm{T}_{\mathrm{M}}^{* 1,0} \oplus \mathrm{T}_{\mathrm{M}}^{* 0,1}, \\
\mathrm{~T}_{\mathrm{M}}^{* 1,0} \oplus \mathrm{T}_{\mathrm{M}}^{* 1,0} \ni\left(\xi_{1}, \xi_{2}\right) \longrightarrow \xi_{1}-\xi_{2} \in \mathbb{C T}_{\mathrm{M}}^{*} .
\end{gathered}
$$

A CR gauge is a bundle map

$$
\lambda: \mathbb{C T}_{\mathrm{M}}^{*} \longrightarrow \mathrm{T}_{\mathrm{M}}^{* 1,0} \text {, such that } \xi-\frac{1}{2} \lambda(\xi) \in \mathrm{T}_{\mathrm{M}}^{* 0,1}, \forall \xi \in \mathbb{C T}_{\mathrm{M}}^{*} .
$$

Note that, in particular,

$$
\lambda\left(\mathrm{T}_{\mathrm{M}}^{* 0,1}\right) \subseteq \mathbb{C H}_{\mathrm{M}}^{0} \quad \text { and } \quad \xi-\frac{1}{2} \lambda(\xi) \in \mathbb{C H}_{\mathrm{M}}^{0}, \quad \forall \xi \in \mathrm{T}_{\mathrm{M}}^{* 1,0} .
$$

Therefore a $C R$ gauge yields a short exact sequence inverting (2.3):

$$
0 \longrightarrow \mathbb{C T}_{\mathrm{M}}^{*} \stackrel{(\lambda / 2) \oplus(\mathrm{id}-\lambda / 2)}{\longrightarrow} \mathrm{T}_{\mathrm{M}}^{* 1,0} \oplus \mathrm{T}_{\mathrm{M}}^{* 0,1} \stackrel{(\lambda / 2-\mathrm{id}, \lambda / 2)}{\longrightarrow} \mathbb{C H}_{\mathrm{M}}^{0} \longrightarrow 0,
$$

where id denotes the identity on $\mathbb{C T}_{\mathrm{M}}^{*}$.

There are several possible choices of $\lambda$. A $C R$ gauge $\lambda$ is said to be of the real type if

$$
\lambda(\xi)+\overline{\lambda(\bar{\xi})}=2 \cdot \xi, \quad \forall \xi \in \mathbb{C T}^{*}{ }_{\mathrm{M}}
$$

and balanced if

$$
\lambda(\xi)=\xi, \quad \forall \xi \in \mathrm{H}_{\mathrm{M}}^{0} .
$$

Existence of balanced $C R$ gauges of the real type was proved in [32, §2A]. The following is then easy to deduce from the above properties.

Proposition 2.2. The bundle map $\lambda$ of a balanced CR gauge

- is a projection of $\mathbb{C T}_{M}^{*}$ onto $\mathrm{T}_{M}^{* 1,0}$; 
- its restriction to $\mathrm{T}_{M}^{*}$ is an isomorphism with a real complement of $i \cdot \mathrm{H}_{M}^{0}$ in $\mathrm{T}_{M}^{* 1,0}$.

Let us define a linear map

$$
\lambda^{p}: \Lambda^{p}\left(\mathbb{C T}_{\mathrm{M}}^{*}\right) \rightarrow \Lambda^{p}\left(\mathrm{~T}_{\mathrm{M}}^{* 1,0}\right)
$$

in such a way that on the monomials we have

$$
\lambda^{p}\left(\xi_{1} \wedge \cdots \wedge \xi_{p}\right)=\lambda\left(\xi_{1}\right) \wedge \cdots \wedge \lambda\left(\xi_{p}\right) .
$$

Proposition 2.3. The map $\lambda^{n+k}$ defines a projection of $\Lambda^{n+k}\left(\mathbb{C T}_{M}^{*}\right)$ onto $K_{M}$, which is independent of the particular choice of a balanced CR gauge $\lambda$ of the real type.

Proof. Fix a point $\mathrm{p}$ of $\mathrm{M}$ and a basis

$$
\xi_{1}, \ldots, \xi_{n}, \tau_{1}, \ldots, \tau_{k}, \zeta_{1}, \ldots, \zeta_{n}
$$

of $\mathbb{C T}_{\mathrm{M}, \mathrm{p}}^{*}$ with $\xi_{i} \in \mathrm{T}_{\mathrm{M}, \mathrm{p}}^{* 1,0}$, for $1 \leq i \leq n, \tau_{j} \in \mathrm{H}_{\mathrm{M}}^{0}$ for $1 \leq j \leq k$ and $\xi_{i} \in \mathrm{T}_{\mathrm{M}, \mathrm{p}}^{* 0,1}$ for $1 \leq i \leq n$.

If $\lambda$ is a balanced $C R$ gauge of the real type, then $\lambda\left(\xi_{i}\right)=\xi_{i}$ for $1 \leq i \leq n$, $\lambda\left(\tau_{j}\right)=\tau_{j}$ for $1 \leq j \leq k, \lambda\left(\zeta_{i}\right) \in \mathbb{C H}_{\mathrm{M}}^{0}$ for $1 \leq i \leq n$. Hence the image by $\lambda^{n+k}$ of all monomials of degree $n+k$ in the elements of the basis (†) which contain a factor $\zeta_{i}$ contain at least $k+1$ factors in $\mathbb{C H}_{\mathrm{M}}^{0}$ and is therefore 0 , while

$$
\lambda^{n+k}\left(\xi_{1} \wedge \cdots \wedge \xi_{n} \wedge \tau_{1} \wedge \cdots \wedge \tau_{k}\right)=\xi_{1} \wedge \cdots \wedge \xi_{n} \wedge \tau_{1} \wedge \cdots \wedge \tau_{k} .
$$

This completes the proof.

Definition 2.2. We call $\lambda^{n+k}$ the natural projection onto the canonical bundle.

2.3. $C R$ maps. Let $\left(\mathrm{M}_{i}, \mathrm{~K}_{\mathrm{M}_{i}}\right)$ be abstract $C R$ manifolds of type $\left(n_{i}, k_{i}\right)(i=1,2)$, $f: \mathrm{M}_{1} \rightarrow \mathrm{M}_{2}$ a smooth map and $f_{*}: \mathbb{C T}_{\mathrm{M}_{1}} \rightarrow \mathbb{C T}_{\mathrm{M}_{2}}$ the bundle map obtained by complexifying its differential.

Definition 2.3. The map $f$ is said to be:

- $C R$ if $f_{*}\left(\mathrm{~T}_{\mathrm{M}_{1}}^{0,1}\right) \subseteq \mathrm{T}_{\mathrm{M}_{2}}^{0,1}$;

- a $C R$ immersion (embedding) if it is a smooth immersion (embedding) and moreover $\mathrm{T}_{\mathrm{M}_{1}}^{0,1}=\left\{Z \in \mathbb{C T}_{\mathrm{M}_{1}} \mid f_{*}(Z) \in \mathrm{T}_{\mathrm{M}_{2}}^{0,1}\right\}$.

- a $C R$ submersion if it is a smooth submersion and $f_{*}\left(\mathrm{~T}^{0,1} \mathrm{M}_{1}\right)=\mathrm{T}^{0,1} \mathrm{M}_{2}$.

A $C R$ immersion (embedding) is called generic when $n_{1}+k_{1}=n_{2}+k_{2}$.

On an abstract $C R$ manifold $\mathrm{M}$ of type $(n, k)$, we consider the Grassmann subalgebra $\Omega_{\mathrm{M}}^{*}$ of $\Lambda^{*}\left(\mathbb{C T}_{\mathrm{M}}^{*}\right)$ generated by $\mathrm{T}_{\mathrm{M}}^{* 1,0}$ :

$$
\Omega_{\mathrm{M}}^{*}=\sum_{p=0}^{n+k} \Omega_{\mathrm{M}}^{p}, \quad \text { with } \quad \Omega_{\mathrm{M}}^{p}=\Omega_{\mathrm{M}}^{*} \cap \Lambda^{p}\left(\mathbb{C T}_{\mathrm{M}}^{*}\right) .
$$

With this notation, $\mathrm{K}_{\mathrm{M}}=\Omega_{\mathrm{M}}^{n+k}$. CR maps and immersion can be described by using the bundles $\Omega_{\mathrm{M}}^{*}$.

Proposition 2.4. Let $M_{i}$ be abstract $C R$ manifolds of type $\left(n_{i}, k_{i}\right)$, for $i=1,2$ and $f: M_{1} \rightarrow M_{2}$ a smooth map. Then $f$ is 
- $C R$ iff $f^{*}\left(\Omega_{M_{2}}^{1}\right) \subseteq \Omega_{M_{1}}^{1}$;

- a CR immersion iff $f$ is a smooth immersion and $f^{*}\left(\Omega_{M_{2}}^{n_{1}+k_{1}}\right)=K_{M_{1}}$. A generic CR-immersion $f: M_{1} \rightarrow M_{2}$ is characterized by

$$
f^{*}\left(K_{M_{2}}\right)=K_{M_{1}} \text {. }
$$

2.4. $q$-pseudoconcavity. Let $q$ be a nonegative integer. Following [21], we say that a $C R$ manifold $M$, of type $(n, k)$, is q-pseudoconcave if, for every $\mathrm{p} \in M$ and every characteristic covector $\xi \in \mathrm{H}_{\mathrm{p}}^{0} M \backslash\{0\}$, the scalar Levi form $\mathscr{L}_{\mathrm{p}}(\xi, \cdot)$ has at least $q$ negative and $q$ positive eigenvalues.

We recall that

$$
\mathscr{L}_{\mathrm{p}}(\xi, v)=\xi\left(\left[\mathrm{J}_{\mathrm{M}} X, X\right]\right)=\mathrm{d} \tilde{\xi}\left(v, \mathrm{~J}_{\mathrm{M}}(v)\right), \text { for } v \in \mathrm{H}_{\mathrm{p}} M,
$$

with $X \in \Gamma^{\infty}\left(M, \mathrm{H}_{\mathrm{M}}\right), \tilde{\xi} \in \Gamma^{\infty}\left(M, \mathrm{H}^{0} M\right)$ and $X_{\mathrm{p}}=v, \tilde{\xi}_{\mathrm{p}}=\xi$.

The $C R$ dimension $n$ of a $q$-pseudoconcave $C R$ manifold is at least $2 q$ (more precise lower bounds on $n$, depending also on the $C R$ codimension $k$, can be obtained e.g. from [7]).

Various equivalent definitions of the Levi form and more of its aspects are explained in [13].

2.5. Deformation of $C R$ structures. With the preparation of $\$ 2.1$, we can define $C R$ deformations of a $C R$ manifold $\mathrm{M}$ of general type $(n, k)$ in terms of canonical bundles, allowing in this way also a deformation of the underlying contact structure $\mathrm{H}_{\mathrm{M}}$.

Definition 2.4. A smooth one-parameter deformation of a $C R$ structure $\mathrm{K}_{\mathrm{M}}$ on $\mathrm{M}$ is a smooth map $\mathrm{M} \times\left(-\epsilon_{0}, \epsilon_{0}\right) \ni(\mathrm{p}, \mathrm{t}) \rightarrow\left[\mathrm{K}_{\mathrm{M}_{\mathrm{t}} \mathrm{p}}\right] \in \mathbb{P}\left(\Lambda^{n+k}\left(\mathbb{C T}^{*}\right)\right)$ such that, for each $\mathrm{t} \in\left(-\epsilon_{0}, \epsilon_{0}\right), \mathrm{K}_{\mathrm{M}_{\mathrm{t}}}$ is the canonical complex line bundle of a $C R$ structure on $\mathrm{M}$ and $\mathrm{K}_{\mathrm{M}_{0}}=\mathrm{K}_{\mathrm{M}}$.

Line bundles over a contractible base are trivial (see e.g. [27, Cor.3.4.8]). Hence, for a compact $\mathrm{M}$, we can find a finite open covering $\mathcal{U}=\left\{U_{j}\right\}$ to describe the deformation by the data of smooth maps

$$
\mathrm{M} \times\left(-\epsilon_{0}, \epsilon_{0}\right) \ni(\mathrm{p}, \mathrm{t}) \rightarrow \omega_{j}(\mathrm{p}, \mathrm{t}) \in \Lambda^{n+k} \mathbb{C} \mathrm{T}^{*} \mathrm{M}
$$

such that $\omega_{j, \mathrm{t}}(\cdot, \mathrm{t}) \in \Gamma^{\infty}\left(U_{j}, \mathrm{~K}_{M_{t}}\right)$ for each $\mathrm{t} \in\left(-\epsilon_{0}, \epsilon_{0}\right)$.

\section{3. $\bar{\partial}_{\mathrm{M} \text {-СономоLOGY }}$}

Let $\mathrm{M}$ be an abstract $C R$ manifold of type $(n, k)$.

The ideal sheaf $\mathcal{I}_{\mathrm{M}}$ of $\mathcal{A}_{\mathrm{M}}$ generated by the germs of smooth sections of $\mathrm{T}_{\mathrm{M}}^{* 1,0}$ is, according to [14], the characteristic system of $\mathrm{T}_{\mathrm{M}}^{0,1}$. Hence, formal integrability is equivalent to

$$
\mathrm{d}\left(\mathcal{I}_{\mathrm{M}}\right) \subseteq \mathcal{I}_{\mathrm{M}}
$$


For nonnegative integers $p$, its exterior powers $\mathcal{I}_{\mathrm{M}}^{p}$ are also d-closed ideals and we can consider the quotients of the de Rham complex:

$$
\bar{\partial}_{\mathrm{M}}: \mathcal{I}_{\mathrm{M}}^{p} / \mathcal{I}_{\mathrm{M}}^{p+1} \longrightarrow \mathcal{I}_{\mathrm{M}}^{p} / \mathcal{I}_{\mathrm{M}}^{p+1}
$$

(we put $\mathcal{I}_{\mathrm{M}}^{0}=\mathcal{A}_{\mathrm{M}}$ ). Since $\mathcal{I}_{\mathrm{M}}^{p}=0$ when $p>n+k$, the map (*) is nontrivial if and only if $0 \leq p \leq n+k$.

The canonical $\mathbb{Z}$-grading of $\mathcal{A}_{\mathrm{M}}$ induces a $\mathbb{Z}$-grading $\mathcal{I}_{\mathrm{M}}^{p}=\bigoplus_{q=0}^{n} \mathcal{I}_{\mathrm{M}}^{p, q}$ of $\mathcal{I}_{\mathrm{M}}^{p}$, with $\mathcal{I}_{\mathrm{M}}^{p, q}=\mathcal{I}_{\mathrm{M}}^{p} \cap \mathcal{A}_{\mathrm{M}}^{p+q}$. Let us set $Q_{\mathrm{M}}^{p \text {,* }}=\mathcal{I}_{\mathrm{M}}^{p} / \mathcal{I}_{\mathrm{M}}^{p+1}$. By passing to the quotients, the $\mathbb{Z}$-gradings of the exterior powers of $\mathcal{I}_{\mathrm{M}}$ induce the $\mathbb{Z}$-gradings

$$
Q_{\mathrm{M}}^{p, *}=\bigoplus_{q=0}^{n} Q_{\mathrm{M}}^{p, q}
$$

For each pair of integers $(p, q)$ with $0 \leq p \leq n+k, 0 \leq q \leq n$ the summand $Q_{M}^{p, q}$ is the sheaf of germs of smooth sections of a complex vector bundle $Q_{M}^{p, q}$ on $M$. Then for each $0 \leq p \leq n+k$, the map $(*)$ is a differential complex

$$
0 \rightarrow Q_{M}^{p, 0} \stackrel{\bar{\partial}_{\mathrm{M}}}{\longrightarrow} Q_{\mathrm{M}}^{p, 1} \longrightarrow \cdots \stackrel{\bar{\partial}_{\mathrm{M}}}{\longrightarrow} Q_{\mathrm{M}}^{p, n-1} \stackrel{\bar{\partial}_{\mathrm{M}}}{\longrightarrow} Q_{\mathrm{M}}^{p, n} \rightarrow 0,
$$

which is called the tangential Cauchy-Riemann complex in degree $p$ (for more details see e.g. [21]).

Since $\mathcal{I}_{\mathrm{M}}^{n+k+1}=\underline{0}$, the bundles $\mathrm{Q}_{\mathrm{M}}^{n+k, q}$ are subbundles of $\Lambda^{n+k+q}\left(\mathbb{C T}_{\mathrm{M}}^{*}\right)$ and in particular $\mathrm{Q}_{\mathrm{M}}^{n+k, 0}$ is the same as the canonical bundle $\mathrm{K}_{\mathrm{M}}$. As we will explain below, it is a $C R$ line bundle and the tangential Cauchy-Riemann complex in degree $n+k$ is also its $\bar{\partial}_{\mathrm{M}}$-complex as a $C R$ line bundle. This is a special example of a notion that was described e.g. in [22, §7] and that we will quickly recall in the next subsection.

The tangential Cauchy-Riemann complexes can be described in a way that is suitable to deal with smooth deformations of the $C R$ structure.

Let us set $\mathrm{T}_{\mathrm{M}}^{* p, 0}=\Lambda^{p}\left(\mathrm{~T}_{\mathrm{M}}^{* 1,0}\right)$ and denote by $\mathcal{T}_{\mathrm{M}}^{* p, 0}$ the sheaf of germs of smooth sections of $\mathrm{T}_{\mathrm{M}}^{* p, 0}$.

3.1. Differential presentation of the tangential $C R$ complex. Let $\mathrm{E}_{\mathrm{M}}$ be a complement of $\mathrm{T}_{\mathrm{M}}^{* 1,0}$ in $\mathbb{C T}_{\mathrm{M}}^{*}$. We can e.g. fix a smooth Hermitian product on the fibers of $\mathbb{C T}_{M}^{*}$ and take $\mathrm{E}_{\mathrm{M}}=\left(\mathrm{T}_{\mathrm{M}}^{* 1,0}\right)^{\perp}$. The fiber bundle $\mathrm{E}_{\mathrm{M}}$ has rank $n$ and we obtain a bigradation of the Grassmann algebra of the complexified cotangent bundle of $\mathrm{M}$ by setting

$$
\Lambda^{*}\left(\mathbb{C T}_{\mathrm{M}}^{*}\right)=\sum_{\substack{0 \leq p \leq n+k \\ 0 \leq q \leq n}} \mathrm{E}_{\mathrm{M}}^{p, q}, \quad \text { with } \mathrm{E}_{\mathrm{M}}^{p, q}=\Lambda^{q}\left(\mathrm{E}_{\mathrm{M}}\right) \otimes_{\mathrm{M}} \mathrm{T}_{\mathrm{M}}^{* p, 0} .
$$

Denote by $\mathcal{E}_{\mathrm{M}}^{p, q}$ the sheaf of germs of smooth sections of $\mathrm{E}_{\mathrm{M}}^{p, q}$.

Lemma 3.1. For every integer $p$ with $0 \leq p \leq n+k$ we have:

(1) $\mathcal{E}_{M}^{p, q} \subseteq \mathcal{I}_{M}^{p}$.

(2) The restriction of the projection onto the quotient defines isomorphisms

$$
E_{M}^{p, q} \rightarrow Q_{M}^{p, q}, \quad \mathcal{E}_{M}^{p, q} \rightarrow Q_{M}^{p, q}
$$


of fiber bundles and of their sheaves of sections.

By using the isomorphisms of (3.1) we can rewrite the tangential CauchyRiemann complexes in the form

$$
0 \longrightarrow \mathcal{E}_{\mathrm{M}}^{p, 0} \stackrel{\mathrm{d}_{\mathrm{M}}^{\prime \prime}}{\longrightarrow} \mathcal{E}_{\mathrm{M}}^{p, 1} \stackrel{\mathrm{d}_{\mathrm{M}}^{\prime \prime}}{\longrightarrow} \cdots \rightarrow \mathcal{E}_{\mathrm{M}}^{p, n-1} \stackrel{\mathrm{d}_{\mathrm{M}}^{\prime \prime}}{\longrightarrow} \mathcal{E}_{\mathrm{M}}^{p, n} \longrightarrow 0 .
$$

We get explicit representations of $\mathrm{d}_{\mathrm{M}}^{\prime \prime}$, and hence of $Q_{\mathrm{M}}^{p, q}$ and $\bar{\partial}_{\mathrm{M}}$, by choosing suitable coframes of $\mathbb{C T}_{\mathrm{M}}^{*}$. Take indeed on an open subset $U$ of $\mathrm{M}$ a coframe $\omega_{1}, \ldots, \omega_{n}, \eta_{1}, \ldots, \eta_{n+k}$ of $\mathrm{T}_{\mathrm{M}}^{*}$

$$
\text { with } \omega_{1}, \ldots, \omega_{n} \text { in } \mathcal{E}_{\mathrm{M}} \text { and } \eta_{1}, \ldots, \eta_{n+k} \in \Gamma^{\infty}\left(U^{\prime}, \mathrm{T}_{\mathrm{M}}^{* 1,0}\right) \text {. }
$$

Then there are uniquely determined smooth complex vector fields $\bar{L}_{1}, \ldots, \bar{L}_{n}$, $P_{1}, \ldots, P_{n+k}$ such that

$$
\mathrm{d} u=\sum_{j=1}\left(\bar{L}_{j} u\right) \omega_{j}+\sum_{i=1}^{n+k}\left(P_{i} u\right) \eta_{i}
$$

The vector fields $\bar{L}_{h}$, for $1 \leq h \leq n$, are characterized by

$$
\omega_{j}\left(\bar{L}_{h}\right)=\delta_{j, h}(\text { Kronecker delta }), \quad 1 \leq j \leq n, \quad \eta_{i}\left(\bar{L}_{h}\right)=0, \quad 1 \leq i \leq n+k,
$$

and hence are sections of $\mathrm{T}_{\mathrm{M}}^{0,1}$. By our definition,

$$
\mathrm{d}_{\mathrm{M}}^{\prime \prime} u=\sum_{j=1}^{n}\left(\bar{L}_{j} u\right) \omega_{j}, \quad \forall u \in C^{\infty}(U)
$$

For each $j$ with $1 \leq j \leq n$ we have

$$
\mathrm{d} \omega_{j}=\sum_{1 \leq j_{1} \leq j_{2} \leq n} c_{j}^{j_{1}, j_{2}} \omega_{j_{1}} \wedge \omega_{j_{2}}+\zeta_{j}
$$

with $c_{j}^{j_{1}, j_{2}} \in C^{\infty}(U), \zeta_{j} \in \mathcal{I}_{\mathrm{M}}(U)$. This gives in particular

$$
\mathrm{d}_{\mathrm{M}}^{\prime \prime} \omega_{j}=\sum_{1 \leq j_{1} \leq j_{2} \leq n} c_{j}^{j_{1}, j_{2}} \omega_{j_{1}} \wedge \omega_{j_{2}}
$$

and hence, computing by recurrence on the number of factors, we get

$$
\begin{gathered}
d_{M}^{\prime \prime}\left(\omega_{j_{0}} \wedge \omega_{j_{1}} \wedge \cdots \wedge \omega_{j_{q}}\right)=d_{M}^{\prime \prime}\left(\omega_{j_{0}}\right) \wedge \omega_{j_{1}} \wedge \cdots \wedge \omega_{j_{q}}+\omega_{j_{0}} \wedge d_{M}^{\prime \prime}\left(\omega_{j_{1}} \wedge \cdots \wedge \omega_{j_{q}}\right), \\
d_{M}^{\prime \prime}\left(\omega_{j_{1}} \wedge \cdots \wedge \omega_{j_{q}} \wedge \eta_{i_{1}} \wedge \cdots \wedge \eta_{i_{p}}\right)=\left(d_{M}^{\prime \prime}\left(\omega_{j_{1}} \wedge \cdots \wedge \omega_{j_{q}}\right)\right) \wedge \eta_{i_{1}} \wedge \cdots \wedge \eta_{i_{p}} .
\end{gathered}
$$

These formulas yield explicit local expressions for $\mathrm{d}_{\mathrm{M}}^{\prime \prime}$. We note that for $p=0$ they only depend on the choice of the coframe $\omega_{1}, \ldots, \omega_{n}$ of $E_{M}$.

In particular, if $\mathrm{M}$ is compact and $\left\{\mathrm{M}_{\mathrm{t}}\right\}_{|\mathrm{t}|<\epsilon_{0}}$ a smooth deformation of its $C R$ structure, then $\mathrm{E}_{\mathrm{M}}$ stays transversal to $\mathrm{T}_{\mathrm{M}_{\mathrm{t}}}^{* 1,0}$ for small $|\mathrm{t}|$ and therefore the tangential Cauchy-Riemann complex in degree 0 for $M_{t}$ can be defined on sections of the same vector bundles for all small $t$.

3.2. $C R$ line bundles. A $C R$ line bundle on $\mathrm{M}$ is the datum of a smooth complex line bundle $\pi: \mathrm{F} \rightarrow \mathrm{M}$, together with a $C R$ structure on $\mathrm{F}$ which is compatible with the linear structure of the fibers and for which $\pi$ is a $C R$ submersion. The compatible $C R$ structures on $\mathrm{F}$ are parametrized (modulo $C R$ equivalence) by special cohomology classes of $H^{1}\left(\mathrm{M}, \mathcal{H}_{\mathrm{M}}^{0,1}\right)$, where $\mathcal{H}_{\mathrm{M}}^{0,1}$ is the sheaf of germs of local cohomology classes of degree $(0,1)$ on $\mathrm{M}$ : In a 
local trivialization $\left.\left\{U_{i}, s_{i}\right)\right\}$ of $\mathrm{F}$, with transition functions $\left(\gamma_{i, j}\right)$, the structure is described by the datum of $\bar{\partial}_{\mathrm{M}}$-closed forms $\phi_{i}$ in $Q_{M}^{0,1}\left(U_{i}\right)$ with

$$
\bar{\partial}_{\mathrm{M}}^{\mathrm{F}} s_{i}=\phi_{i} \otimes s_{i} \text { on } U_{i} \text { and } \phi_{i}-\phi_{j}=\gamma_{i, j}^{-1} \bar{\partial}_{\mathrm{M}} \gamma_{i, j} \text { on } U_{i} \cap U_{j} \text {. }
$$

We can consistently define a differential operator $\bar{\partial}_{\mathrm{M}}^{\mathrm{F}}$ on smooth sections of $\mathrm{F}$, with values in the smooth sections of $\mathrm{Q}_{\mathrm{M}}^{0,1} \otimes \mathrm{F}$, in such a way that

$$
\bar{\partial}_{\mathrm{M}}^{\mathrm{F}}\left(f s_{i}\right)=\left(\bar{\partial}_{\mathrm{M}} f \otimes s_{i}\right)+\left(f \cdot \phi_{i}\right) \otimes s_{i}, \quad \forall f \in C^{\infty}\left(U_{i}\right) .
$$

Let us use the notation $\mathcal{F}_{M}$ to indicate the sheaf of germs of smooth sections of $F_{M}$ and $\mathcal{F}_{M}^{p, q}$ for the sheaf of germs of smooth sections on $\mathrm{M}$ of the bundle $\mathrm{Q}_{\mathrm{M}}^{p, q} \otimes \mathrm{F}$. Then we can define, in general, a differential operator $\bar{\partial}_{\mathrm{M}}^{\mathrm{F}}$ mapping sections of $\mathcal{F}_{\mathrm{M}}^{p, q}$ into sections of $\mathcal{F}_{\mathrm{M}}^{p, q+1}$ by setting for $U$ open in $\mathrm{M}$, $\alpha \in Q_{\mathrm{M}}^{p, q}(U)$ and $\sigma \in \mathcal{F}_{\mathrm{M}}(U)$,

$$
\bar{\partial}_{\mathrm{M}}^{\mathrm{F}}(\alpha \otimes \sigma)=\left(\bar{\partial}_{\mathrm{M}} \alpha\right) \otimes \sigma+(-1)^{p+q} \alpha \wedge \partial_{\mathrm{M}}^{\mathrm{F}} \sigma .
$$

In this way we obtain the tangential Cauchy-Riemann complexes with coefficients in $F$ :

$$
\begin{aligned}
0 \longrightarrow \mathcal{F}_{\mathrm{M}}^{p, 0}(U) \stackrel{\bar{\partial}_{\mathrm{M}}^{\mathrm{F}}}{\longrightarrow} \mathcal{F}_{\mathrm{M}}^{p, 1}(U) \longrightarrow & \longrightarrow \\
\cdots & \longrightarrow \mathcal{F}_{\mathrm{M}}^{p, n-1}(U) \stackrel{\bar{\partial}_{\mathrm{M}}^{\mathrm{F}}}{\longrightarrow} \mathcal{F}_{\mathrm{M}}^{p, n}(U) \longrightarrow 0 .
\end{aligned}
$$

We denote its cohomology groups by

$$
H^{p, q}\left(U, \mathrm{~F}_{\mathrm{M}}\right)=\frac{\operatorname{ker}\left(\bar{\partial}_{\mathrm{M}}^{\mathrm{F}}: \mathcal{F}_{\mathrm{M}}^{p, q}(U) \rightarrow \mathcal{F}_{\mathrm{M}}^{p, q+1}(U)\right)}{\operatorname{image}\left(\bar{\partial}_{\mathrm{M}}^{\mathrm{F}}: \mathcal{F}_{\mathrm{M}}^{p, q-1}(U) \rightarrow \mathcal{F}_{\mathrm{M}}^{p, q}(U)\right)} .
$$

When the $C R$ structure on $\mathrm{M}$ is induced from a generic embedding into an $(n+k)$-dimensional complex manifold $\mathrm{X}$, the $C R$ line bundle $\mathrm{K}_{\mathrm{M}}$ is the restriction to $\mathrm{M}$ of the holomorphic canonical line bundle $\mathrm{K}_{\mathrm{X}}$ of $\mathrm{X}$. In general, the canonical bundle of an abstract $C R$ manifold might not even be locally $C R$ trivializable, as explained e.g. in [9, 22, 28].

For a $C R$ submanifold $\mathrm{M}$ of an $\mathrm{N}$-dimensional projective space $\mathbb{C P}^{\mathrm{N}}$, the restrictions $\mathscr{O}_{\mathrm{M}}(-1)$ and $\mathscr{O}_{\mathrm{M}}(1)$ to $\mathrm{M}$ of its tautological and hyperplane bundles are $C R$ line bundles on $\mathrm{M}$, dual to each other. By the Euler sequence, the canonical bundle $\mathrm{K}_{\mathbb{C P}^{N}}$ of the projective space is isomorphic to the $(\mathrm{N}+1)$ th power $\mathscr{O}_{\mathbb{C P}^{\mathrm{N}}}(-\mathrm{N}-1)$ of the tautological bundle (see e.g. [19, p.146]) and therefore its dual $\mathrm{K}_{\mathrm{Cp}^{N}}^{*}$, being isomorphic to the $(\mathrm{N}+1)$-th power $\mathscr{O}_{\mathbb{C P}^{\mathrm{N}}}(\mathrm{N}+1)$ of the hyperplane bundle $\mathscr{O}_{\mathbb{C P}^{\mathrm{N}}}(1)$, which is very ample, is very ample. In particular, if $\mathrm{M}$ is a generic $C R$ submanifold of $\mathbb{C P}^{\mathbb{N}}$, then its anti-canonical bundle $\mathrm{K}_{\mathrm{M}}^{*}$ is the restriction to $\mathrm{M}$ of a very ample line bundle. If $\mathrm{E}_{\mathrm{X}}$ is a very ample line bundle on a complex compact complex manifold $\mathrm{X}$, then its

\footnotetext{
${ }^{2}$ This is obtained by identifying $\mathrm{F}$ with its vertical bundle and first defining $\bar{\partial}_{\mathrm{M}}^{\mathrm{F}}$ on the smooth local sections $s$ of $\mathrm{F}$ by setting

$$
\bar{\partial}_{\mathrm{M}}^{\mathrm{F}} s\left(X+i J_{\mathrm{M}} X\right)=d s(X)+J_{\mathrm{F}} d s\left(J_{\mathrm{M}} X\right), \quad \forall X \in H M .
$$

In fact, when $s$ is a section, the right hand side of $(\dagger)$ is vertical.
} 
first Chern class $c_{1}\left(E_{X}\right)$ is positive and the Kodaira-Nakano theorem tells us that this property is sufficient to ensure that the cohomology groups $H^{q}\left(\mathrm{X}, \mathrm{K}_{\mathrm{X}} \otimes \mathrm{E}_{\mathrm{X}}\right)$ vanish for $q>0$ (see e.g. [19, p.154]). On the other hand, it is known that also the anti-canonical bundles of the generalized complex flag manifolds are positive (see e.g. [6, 8]). They are therefore examples of the larger class of Fano varieties, which are characterized by the ampleness of their anti-canonical bundle $\mathrm{K}_{\mathrm{X}}^{*}$.

When $X$ is a flag manifold of a complex semisimple Lie group $\mathbf{G}$, the minimal orbits in $X$ of its real forms $\mathbf{G}_{\mathbb{R}}$, studied e.g. in [3, 37], are examples of generic $C R$ submanifolds of Fano varieties.

3.3. Deformations and $C R$ line bundles. A Cartier divisor in $\mathrm{X}$ is the effective divisor of a section of a line bundle $F_{X}$ on $X$. A positive $F_{X}$ has a power which is very ample and therefore the support of its Cartier divisor is the support of the pole divisor of a global meromorphic function $f$ on $\mathrm{X}$.

Let $\mathrm{X}$ be a complex space, $f$ a global meromorphic function on $\mathrm{X}$. The corresponding complex line bundle $F_{X}$ can be described in the following way: Let $\left\{U_{i}\right\}$ be an open covering of $\mathrm{X}$ by connected open subsets with the property that, for each index $i$, there are relatively prime $f_{i}^{\prime}, f_{i}^{\prime \prime} \in \mathscr{O}_{\mathbf{X}}\left(U_{i}\right)$ such that $f_{i}^{\prime} / f_{i}^{\prime \prime}=f$ on $U_{i}$. The quotients $f_{i}^{\prime} / f_{j}^{\prime}=f_{i}^{\prime \prime} / f_{j}^{\prime \prime}=g_{i, j}$ define nowhere vanishing holomorphic functions on $U_{i} \cap U_{j}$, which are the transition functions of a holomorphic line bundle $\mathrm{F}_{\mathrm{X}}$ on the trivialization atlas $\left\{U_{i}\right\}$. Then

$$
D=\bigcup_{i}\left\{\mathrm{p} \in U_{i} \mid f_{i}^{\prime \prime}(\mathrm{p})=0\right\}
$$

is the support of the pole divisor of $f$.

Proposition 3.2. Let $X$ be a complex space, $D$ the support of the pole divisor of a global meromorphic function $f$ on $X$ and $F_{X}$ the corresponding line bundle. Let $M$ be a smooth $C R$ submanifold of $X$ and $\left\{M_{t}\right\}$ a deformation on $M$ of the CR structure $M_{0}$ induced on $M$ by $X$ such that for each the $C R$ structures of $M_{t}$ and $M_{0}$ agree to infinite order on $D \cap M$. Assume that the $C R$ embedding $M_{0} \hookrightarrow X$ is generic. Then we can define $C R$ line bundles $F_{M_{t}}$ on each $M_{t}$ in such a way that $\left\{F_{M_{t}}\right\}$ is a smooth deformation of $F_{M}$, and their CR structures agree to infinite order on the fibers over D.

Proof. We keep the notation introduced at the beginning of the subsection. The restrictions of the transition functions $g_{i, j}$ define a $C R$ line bundle on $M_{0}$, because

$$
g_{i, j}^{-1} \bar{\partial}_{M} g_{i, j}=0 \text { on } U_{i} \cap U_{j} \cap \mathrm{M} .
$$

Let us consider now an element of the deformation $\left\{\mathrm{M}_{\mathrm{t}}\right\}$. We note that $g_{j, i} \bar{\partial}_{\mathrm{M}_{\mathrm{t}} g_{i, j}}=\frac{f_{j}^{\prime \prime}}{f_{i}^{\prime \prime}} \frac{f_{j}^{\prime \prime} \bar{\partial}_{\mathrm{M}_{\mathrm{t}}} f_{i}^{\prime \prime}-f_{i}^{\prime \prime} \bar{\partial}_{\mathrm{M}_{\mathrm{t}}} f_{j}^{\prime \prime}}{f_{j}^{\prime \prime 2}}=\frac{\bar{\partial}_{\mathrm{M}_{\mathrm{t}}} f_{i}^{\prime \prime}}{f_{i}^{\prime \prime}}-\frac{\bar{\partial}_{\mathrm{M}_{\mathrm{t}}} f_{j}^{\prime \prime}}{f_{j}^{\prime \prime}}, \quad$ on $U_{i} \cap U_{j} \cap \mathrm{M}$ 
is the difference of two smooth $\bar{\partial}_{\mathrm{M}_{\mathrm{t}}}$-closed forms, the first one defined on $U_{i} \cap \mathrm{M}$, the second on $U_{j} \cap \mathrm{M}$. Indeed

$$
\phi_{i}^{(\mathrm{t})}= \begin{cases}f_{i}^{\prime \prime-1} \cdot \bar{\partial}_{\mathrm{M}_{\mathrm{t}}} f_{i}^{\prime \prime}, & \text { on }\left(U_{i} \cap \mathrm{M}\right) \backslash D, \\ 0, & \text { on } U_{i} \cap \mathrm{M} \cap D,\end{cases}
$$

is a smooth $\bar{\partial}_{\mathrm{M}_{\mathrm{t}}}$-closed $(0,1)$ form on $U_{i} \cap \mathrm{M}$, because $\bar{\partial}_{\mathrm{M}_{\mathrm{t}}} f_{i}^{\prime \prime}$ vanishes to infinite order on $D \cap U_{i} \cap \mathrm{M}$ by the assumption that the $C R$ structures of M and $\mathrm{M}_{\mathrm{t}}$ agree to infinite order on $D \cap \mathrm{M}$, while $f_{i}^{\prime \prime}$, which extends holomorphically to the neighourhood $U_{i}$ of $U_{i} \cap \mathrm{M}$ in $\mathrm{X}$, cannot vanish to infinite order at any point of $D \cap \mathrm{M}$.

The complex smooth line bundle $\mathrm{F}_{\mathrm{M}}$ is obtained by gluing the trivial line bundles $\left\{U_{i} \times \mathbb{C}\right\}$ by the transition functions $\left\{g_{i, j}\right\}$. Let $\sigma_{i}$ be the section $U_{i} \times\{1\}$ on $U_{i}$. The $C R$ structure on $\mathrm{F}_{\mathrm{M}_{\mathrm{t}}}$ is obtained by setting

$$
\bar{\partial}_{\mathrm{M}_{\mathrm{t}}}^{\mathrm{F}} \sigma_{i}=\phi_{i}^{(\mathrm{t})} \cdot \sigma_{i} \quad \text { on } U_{i} \text { for all } i
$$

Clearly it agrees to infinite order on $D$ with that of $\mathrm{F}_{\mathrm{M}_{0}}$.

\section{An EXAmple}

Let $m$ be an integer $\geq 2$ and $\mathrm{M}$ the quadric hypersurface of $\mathbb{C P}^{m}$ described in homogeneous coordinates by

$$
\mathrm{M}=\left\{z_{0} \bar{z}_{0}+z_{1} \bar{z}_{1}=z_{2} \bar{z}_{2}+\cdots+z_{m} \bar{z}_{m}\right\} .
$$

The rational function $\zeta_{1}=z_{1} / z_{0}$ has divisor $\left(\zeta_{1}\right)=D_{1}-D_{0}$ with

$$
D_{0}=\left\{z_{0}=0\right\}, \quad D_{1}=\left\{z_{1}=0\right\} \quad \text { and } D_{1} \cap D_{2} \cap \mathrm{M}=\emptyset .
$$

Since the point $[1,0, \ldots, 0]$ does not belong to $M$, the projection map

$$
\pi: \mathbb{C P}^{m} \backslash\{[1,0, \ldots, 0]\} \ni\left[z_{0}, z_{1}, \ldots, z_{m}\right] \longrightarrow\left[z_{1}, \ldots, z_{m}\right] \in \mathbb{C P}^{m-1}
$$

is well defined on $M$. Its image is the complement in $\mathbb{C P}^{m-1}$ of the open ball

$$
\mathrm{B}=\left\{z_{2} \bar{z}_{2}+\cdots+z_{m} \bar{z}_{m}<z_{1} \bar{z}_{1}\right\}
$$

Let $F$ be a smooth function on $\mathbb{C P}^{m-1}$ which is holomorphic on $\mathrm{B}$ and $\mathrm{q}$ any point of its boundary $\partial \mathrm{B}$. If we can find an open neighbourhood $V$ of q in $\mathbb{C P}^{m-1}$ and a solution $v$ to the Cauchy problem

$$
\begin{cases}v \in C^{\infty}(V \backslash \overline{\mathrm{B}}) \cap C^{0}(V \backslash \mathrm{B}), \\ \bar{\partial} v=\bar{\partial} F, & \text { on } V \backslash \overline{\mathrm{B}}, \\ v=0, & \text { on } V \cap \partial \mathrm{B},\end{cases}
$$

then

$$
F^{\prime}= \begin{cases}F, & \text { on } V \cap \mathrm{B}, \\ F-v, & \text { on } V \backslash \mathrm{B}\end{cases}
$$

continues analytically $\left.F\right|_{\mathrm{B}}$ to the point q.

There are holomorphic functions on B which can be continued smoothly, but not analytically, to all points of $\partial \mathrm{B}$. Thus we can find $F \in C^{\infty}\left(\mathbb{C P}^{m-1}\right)$, holomorphic on $\mathrm{B}$, such that $\left.F\right|_{\mathrm{B}}$ has no analytic continuation to any point 
of $\partial \mathrm{B}$ and moreover, since $\partial \mathrm{B} \cap\left\{z_{1}=0\right\}=\emptyset$, we can require that its support does not intersect the hyperplane $\left\{z_{1}=0\right\}$. Then $\alpha=\bar{\partial} F$ is a smooth $(0,1)$ form on $\mathbb{C P}^{m-1}$ with the properties:

- $\bar{\partial} \alpha=0$ and $\operatorname{supp}(\alpha) \subset \mathbb{C P}^{m-1} \backslash \mathrm{B}, \operatorname{supp}(\alpha) \cap\left\{z_{1}=0\right\}=\emptyset$;

- If $V$ is any open neighourhood of a point $\mathrm{q} \in \partial \mathrm{B}$ and $v \in C^{\infty}(V \backslash \overline{\mathrm{B}}) \cap C^{0}(V \backslash \mathrm{B})$ satisfies $\bar{\partial} v=\alpha$ on $V \backslash \overline{\mathrm{B}}$, then $v$ is not 0 at some point of $V \cap \partial \mathrm{B}$.

Let us fix a $(0,1)$ form $\alpha$ on $\mathbb{C P}^{m-1}$ with these properties.

We consider the coordinate charts $\left(U_{0}, \zeta\right),\left(U_{1}, \xi\right)$ in $\mathbb{C P}^{m}$ with

$$
\begin{aligned}
& U_{0}=\left\{z_{0} \neq 0\right\}=\mathbb{C P}^{m} \backslash D_{0}, \quad \zeta_{1}=\frac{z_{1}}{z_{0}}, \xi_{2}=\frac{z_{1}}{z_{0}}, \ldots, \xi_{m}=\frac{z_{m}}{z_{0}}, \\
& U_{1}=\left\{z_{1} \neq 0\right\}=\mathbb{C P}^{m} \backslash D_{1}, \quad \xi_{1}=\frac{z_{0}}{z_{1}}, \xi_{2}=\frac{z_{2}}{z_{1}}, \ldots, \xi_{m}=\frac{z_{m}}{z_{1}} .
\end{aligned}
$$

The pullback $\pi^{*} \alpha$ vanishes to infinte order on the points of $\mathrm{M} \cap D_{0}$, which are mapped by $\pi$ onto the boundary of $\mathrm{B}$. Then $\zeta_{1} \cdot \pi^{*} \alpha$, defined on $\mathrm{M} \backslash D_{0}$, extends to a smooth $(0,1)$ form $\beta$ on $M$, vanishing to infinite order on $\mathrm{M} \cap D_{0}$, whose support is contained in $U_{1} \cap \mathrm{M}$.

We can define a smooth $C R$ structure $\mathrm{M}_{\mathrm{t}}$ on $\mathrm{M}$ by requiring that its canonical bundle has sections

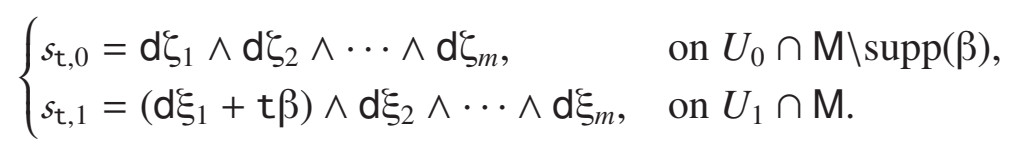

Note that

$$
\begin{aligned}
\mathrm{d} s_{\mathrm{t}, 1} & =\mathrm{t}\left(-\frac{\mathrm{d} \xi_{1}}{\xi_{1}^{2}} \wedge \pi^{*}(\alpha)+\frac{1}{\xi} \pi^{*}(\mathrm{~d} \alpha)\right) \wedge \mathrm{d} \xi_{2} \wedge \cdots \wedge \mathrm{d} \xi_{m} \\
& =-\mathrm{t} \frac{\mathrm{d} \xi_{1}}{\xi_{1}} \wedge \beta \wedge \mathrm{d} \xi_{2} \wedge \cdots \wedge \mathrm{d} \xi_{m}=\frac{\mathrm{t}}{\xi_{1}} \beta \wedge s_{\mathrm{t}, 1}
\end{aligned}
$$

because

$$
\left\{\begin{aligned}
\pi^{*}(d \alpha) \wedge d \xi_{2} \wedge \cdots \wedge d \xi_{m} & =\pi^{*}\left(d \alpha \wedge d \xi_{2} \wedge \cdots \wedge d \xi_{m}\right) \\
= & \pi^{*}\left((\bar{\partial} \alpha) \wedge d \xi_{2} \wedge \cdots \wedge d \xi_{m}\right)=0 .
\end{aligned}\right.
$$

Note that the form $\beta / \xi_{1}=\pi^{*} \alpha / \xi_{1}^{2}$ extends to a smooth form $\beta^{\prime}$, defined on $\mathrm{M}$ and vanishing to infinite order on $D_{0}$, so that

（) $\quad \mathrm{d}_{\mathrm{t}_{\mathrm{t}, 0}}=0$ on $U_{0} \cap \mathrm{M} \backslash \operatorname{supp}(\beta)$ and $\mathrm{d} s_{\mathrm{t}, 1}=\mathrm{t} \cdot \beta^{\prime} \wedge s_{\mathrm{t}, 1}$ on $U_{1} \cap \mathrm{M}$

shows that the line bundles $\mathrm{K}_{\mathrm{M}_{\mathrm{t}}}$ define formally integrable complex valued distributions.

These $C R$ structures agree to infinite order on $\mathrm{M} \cap D_{0}$ with the $C R$ structure $\mathrm{M}_{0}$ on $\mathrm{M}$ which is induced by its embedding into $\mathbb{C P}^{m}$

Their canonical bundles are isomorphic complex line bundles, since the transition functions are in all cases $g_{0,1}=s_{\mathrm{t}, 0} / s_{\mathrm{t}, 1}=-\xi_{1}^{m+1}=-\xi_{1}^{-m-1}, g_{1,0}=g_{0,1}^{-1}$ on $\mathrm{M} \backslash\left(D_{0} \cup D_{1} \cup \operatorname{supp}(\beta)\right)$.

We note that $\xi_{2}, \ldots, \xi_{m}$ are $C R$ functions on $\mathrm{M}_{\mathrm{t}} \backslash D_{0}$ for all $\mathrm{t}$. A necessary condition for $\mathrm{M}_{\mathrm{t}}$ being locally embeddable at a point $\mathrm{p}$ of $D_{0}$ is that 
one could find an open neighbourhood $U^{\prime}$ of p in $U_{1} \cap \mathrm{M}$ and a function $u \in C^{\infty}\left(U^{\prime}\right)$ such that

$$
\mathrm{d}\left(e^{u} s_{\mathrm{t}, 1}\right)=0 \text { on } U^{\prime} .
$$

This equation can be rewritten, by factoring out $e^{u} \zeta_{1}$ and taking into account $(\dagger)$, as

$$
\left(\xi_{1} \mathrm{~d} u+\frac{\mathrm{t}}{\xi_{1}} \pi^{*} \alpha\right) \wedge \mathrm{d} \xi_{2} \wedge \cdots \wedge \mathrm{d} \xi_{m}=0 \text { on } U^{\prime} \backslash D_{0} .
$$

The fibers of $\pi$ above points of $\mathbb{C P}^{m-1} \backslash \bar{B}$ are circles and reduce to single points over $\partial \mathrm{B}$. Since the projection $\pi$ is proper, by shrinking we may assume that $\pi^{-1}\left(\pi\left(U^{\prime}\right)\right)=U^{\prime}$. By integrating over the fibers we define

$$
v(\mathrm{q})=\frac{-1}{2 \pi i} \oint_{\pi^{-1}(\mathrm{q})} u \xi_{1} d \xi_{1}, \text { for } \mathrm{q} \in \pi\left(U^{\prime}\right) \backslash \partial \mathrm{B} .
$$

This is a smooth function on $\pi\left(U^{\prime}\right) \backslash \partial \mathrm{B}$, which extends to a continuous function, that we still denote by $v$, vanishing on $\pi\left(U^{\prime}\right) \cap \partial \mathrm{B}$. Then from $(*)$ we obtain that

$$
\mathrm{d} v \wedge \mathrm{d} \xi_{2} \wedge \cdots \wedge \mathrm{d} \xi_{m}=\mathrm{t} \alpha \wedge \mathrm{d} \xi_{2} \wedge \cdots \wedge \mathrm{d} \xi_{m}, \quad \text { on } \pi\left(U^{\prime}\right) \backslash \overline{\mathrm{B}} .
$$

This is equivalent to

$$
\bar{\partial} v=\mathrm{t} \alpha, \quad \text { on } \pi\left(U^{\prime}\right) \backslash \overline{\mathrm{B}}
$$

and hence contradicts the choice of $\alpha$ if $t \neq 0$, proving that $M_{t}$ cannot be locally embedded at any point $\mathrm{p} \in D_{0}$ if $\mathrm{t} \neq 0$.

This example from [24] shows that we can construct a smooth deformation $\left\{\mathrm{M}_{\mathrm{t}}\right\}$ of the $C R$ structure $\mathrm{M}_{0}$ of the quadric $\mathrm{M}$ such that no $\mathrm{M}_{\mathrm{t}}$ can be locally embedded into a complex manifold at any point of $D_{0}$ if $t \neq 0$.

The $C R$ structure of the $\mathrm{M}_{\mathrm{t}}$ 's are of type $(m-1,1)$ and $\mathrm{M}_{0}$ is 1 -pseudoconcave. Hence the example shows that Theorem 1.1 cannot be valid if we only require $\mathrm{M}_{0}$ being 1-pseudoconcave.

Remark 4.1. Equations ( $\ddagger$ ) is of the type of those we need to solve to construct projective embeddings in $\$ 6$. It can be rewritten as

$$
\left\{\begin{array}{l}
\bar{\partial}_{\mathrm{M}_{\mathrm{t}}^{\mathrm{K}} s_{\mathrm{t}, 0}}^{\mathrm{K}}=0 \\
\bar{\partial}_{\mathrm{M}_{\mathrm{t}}}^{\mathrm{K}} s_{\mathrm{t}, 1}=\mathrm{t} \cdot \beta^{\prime} s_{\mathrm{t}, 1}
\end{array}\right.
$$

We also have

Then we obtain

$$
\bar{\partial}_{\mathrm{M}_{\mathrm{t}}} u=\bar{\partial}_{\mathrm{M}_{0}} u-\mathrm{t} \cdot \frac{\partial u}{\partial \xi_{1}} \beta
$$

$$
\bar{\partial}_{\mathrm{M}_{\mathrm{t}}}^{\mathrm{K}}\left(\frac{1}{\xi_{1}} s_{\mathrm{t}, 1}^{-1}\right)=-\mathrm{t}\left(\frac{1}{\xi_{1}^{2}} \beta+\frac{1}{\xi_{1}} \beta^{\prime}\right) s_{\mathrm{t}, 1}^{-1}=-2 \frac{\mathrm{t}}{\xi_{1}^{3}} \pi^{*} \alpha s_{\mathrm{t}, 1}^{-1} .
$$

An argument similar to the one used above shows that the equation

$(* *) \quad \bar{\partial}\left(w \cdot s_{\mathrm{t}, 1}^{-1}\right)=\left(\bar{\partial}_{\mathrm{M}_{0}} w-\mathrm{t} \frac{\partial w}{\partial \xi_{1}} \beta-\mathrm{t} \cdot w \beta^{\prime}\right) s_{\mathrm{t}, 1}^{-1}=-2 \frac{\mathrm{t}}{\xi_{1}^{3}} \pi^{*} \alpha s_{\mathrm{t}, 1}^{-1}$ 
has no smooth solution on M. Indeed $(* *)$ can be rewritten in the form

$$
\left(\mathrm{d} w-\frac{\mathrm{t}}{\xi_{1}^{2}} \frac{\partial\left(\xi_{1} w\right)}{\partial \xi_{1}} \pi^{*} \alpha+\frac{2 \mathrm{t}}{\xi_{1}^{3}} \pi^{*} \alpha\right) \mathrm{d} \xi_{2} \wedge \cdots \wedge \mathrm{d} \xi_{m}=0
$$

Multiplying by $\xi_{1}^{2}$ and integrating on the fiber, we see that the term in the middle vanishes, while

$$
v(\mathrm{q})=\frac{-1}{4 \pi i} \oint_{\pi^{-1}(\mathrm{q})}\left(\xi_{1}^{2} w\right) \mathrm{d} \xi_{1}
$$

yields a solution in $C^{\infty}\left(\mathbb{C P}^{m-1} \backslash \overline{\mathrm{B}}\right) \cap C^{0}\left(\mathbb{C P}^{m-1} \backslash \mathrm{B}\right)$, vanishing on $\partial \mathrm{B}$, of the equation $\bar{\partial} v=\alpha$ on $\mathbb{C P}^{m-1} \backslash \overline{\mathrm{B}}$, contradicting the choice of $\alpha$.

This adds a further argument for the need of conditions stronger than 1-pseudoconcavity for the validity of the conclusions of Theorem 1.1 ,

\section{VANISHING RESULTS}

We keep the notation introduced in $\$ 2$, $\$$.

5.1. Embedded $C R$ manifolds. We have the following vanishing result:

Theorem 5.1. Assume that $M$ is a 2-pseudoconcave generic smooth $C R$ submanifold of type $(n, k)$ of a complex variety $X$. Let $F_{X}$ be a positive line bundle on $X$ and $F_{M}$ its restriction to $M$. Then we can find a positive integer $\ell_{0}$ such that

$$
H^{p, q}\left(M, F_{M}^{-\ell}\right)=0, \quad \forall \ell \geq \ell_{0}, 0 \leq p \leq n+k, q=0,1 .
$$

Proof. We know from [21] that a generic 2-pseudoconcave $\mathrm{M}$ has a fundamental system of tubular neighborhoods $U$ in $\mathrm{X}$ that are $(n-2)$-pseudoconvex and $(n+k-2)$-pseudoconcave in the sense of Andreotti-Grauer 3 and for which, for all $0 \leq p \leq n+k$, the natural restriction maps

$$
H^{q}\left(U, \Omega^{p}\left(\mathrm{~F}_{U}\right)\right) \longrightarrow H^{q}\left(M, \Omega^{p}\left(\mathrm{~F}_{\mathrm{M}}\right)\right), \quad q=0,1,
$$

are isomorphisms for any holomorphic line bundle $\mathrm{F}_{U}$ over $U$.

This was proved in [21, Thm.4.1] for the trivial line bundle. The arguments of the proof given there apply also in general, yielding the isomorphism for arbitrary holomorphic vector bundles. The statement is then a consequence of [5, Prop.27], because $U$ is strongly $(n+k-2)$-concave in the sense of Andreotti-Grauert and hence also $\mathrm{F}_{U}^{-1}:=\left.\mathrm{F}_{\mathrm{X}}^{-1}\right|_{U}$ is strongly $(n+k-2)$ concave as a complex space, according to [5] and therefore there is a positive integer $\ell_{0}$ such that $H^{p, q}\left(U, \mathrm{~F}_{\mathrm{X}}^{-\ell}\right)=0$ for $\ell \geq \ell_{0}, 0 \leq p \leq n+k$ and $q=0,1$.

\footnotetext{
${ }^{3}$ A real valued smooth function $\phi$ on an $\mathrm{N}$-dimensional complex manifold $\mathrm{X}$ is strongly $q$-pseudoconvex in the sense of [5] at points where its complex Hessian has at least $(\mathrm{N}-q+1)$ positive eigenvalues. Then $\mathrm{X}$ is called strictly q-pseudoconvex if there is an exhaustion function $\phi \in C^{\infty}(X, \mathbb{R})$ which is strictly $q$-pseudoconvex outside a compact subset of $X$ and strictly $q$-pseudoconcave if there is an exhaustion function $\phi \in C^{\infty}(X, \mathbb{R})$ such that $(-\phi)$ is strictly $q$-pseudoconvex outside a compact subset of $\mathrm{X}$.
} 
5.2. Some estimates for abstract $C R$ manifolds. Let $\mathrm{M}$ be an abstract compact $C R$ manifold of type $(n, k)$ and $\mathrm{F}_{\mathrm{M}}$ a complex $C R$ line bundle on $\mathrm{M}$. To discuss its cohomology groups $H^{p, q}\left(\mathrm{M}, \mathrm{F}_{\mathrm{M}}\right)$ by using functional analytic methods, it is convenient to fix a smooth Riemannian metric on $\mathrm{M}$ and a smooth Hermitian norm on the fibers of $F_{M}$. In this way we are able, for each real $r \geq 0$, to define in a standard way on the smooth sections of $Q_{M}^{p, q} \otimes F_{M}$ the Sobolev $\mathrm{W}^{r}$-norm, involving the $L^{2}$-norms of their fractional derivatives up to order $r$. The completions of $\mathcal{F}^{p, q}(\mathrm{M})$ with respect to these norms are Hilbert spaces, that we denote by $\mathrm{W}^{r}\left(\mathrm{M}, \mathrm{Q}_{\mathrm{M}}^{p, q} \otimes \mathrm{F}_{\mathrm{M}}\right)$. We use the notation

$$
\left(v_{1} \mid v_{2}\right)_{r} \text { and }\|v\|_{r}=\sqrt{(v \mid v)_{r}}, \quad \text { for } v_{1}, v_{2}, v \in \mathrm{W}^{r}\left(\mathrm{M}, \mathrm{Q}_{\mathrm{M}}^{p, q} \otimes \mathrm{F}_{\mathrm{M}}\right)
$$

for their Hermitian scalar products and norms (see e.g. [20]).

In [21] subelliptic estimates were proved for the $\bar{\partial}_{\mathrm{M}}$ complex under pseudoconcavity conditions. Since the arguments are local, they trivially extend to forms with coefficients in a complex $C R$ line bundle. We have in particular the following statement.

Proposition 5.2. Let $M$ be a compact smooth abstract $C R$ manifold of type $(n, k)$ and $F_{M}$ any complex $C R$ line bundle on $M$. Assume that $M$ is 2-pseudoconcave. Then, for every $0 \leq p \leq n+k$ and every real $r \geq 0$ there is a positive constant $c_{r, p}>0$ such that

$$
\left\{\begin{array}{l}
c_{r, p} \cdot\|u\|_{r+(1 / 2)} \leq\left\|\bar{\partial}_{M}^{F} u\right\|_{r}^{2}, \quad \forall u \in \mathcal{F}^{p, 0}(M), \\
c_{r, p} \cdot\|v\|_{r+(1 / 2)} \leq\left\|\bar{\partial}_{M}^{F} v\right\|_{r}^{2}+\left\|\left(\bar{\partial}_{M}^{F}\right)^{*} v\right\|_{r}^{2}+\|v\|_{0}^{2}, \quad \forall v \in \mathcal{F}^{p, 1}(M) .
\end{array}\right.
$$

Let us define the subspace

$$
\mathcal{N}_{\mathrm{F}}^{p, 1}(\mathrm{M})=\left\{v \in \mathcal{F}^{p, 1}(\mathrm{M}) \mid \bar{\partial}_{\mathrm{M}}^{\mathrm{F}} v=0, \quad\left(\bar{\partial}_{\mathrm{M}}^{\mathrm{F}}\right)^{*} v=0\right\} .
$$

Keeping the assumptions and the notation above, we have

Proposition 5.3. Under the assumptions of Proposition 5.2.

- The space $\mathcal{N}_{F}^{p, 1}(M)$ is finite dimensional and equals its weak closure with respect to the $L^{2}$ norm;

- For every pair of real numbers $r, r^{\prime}$ with $-(1 / 2) \leq r^{\prime}<r, r \geq 0$, there is a constant $C_{r, r^{\prime}, p}>0$ such that

$$
\left\{\begin{aligned}
\|v\|_{r^{\prime}+(1 / 2)} \leq C_{r, r^{\prime} p}\left(\left\|\bar{\partial}_{M}^{F} v\right\|_{r}^{2}+\left\|\left(\bar{\partial}_{M}^{F}\right)^{*} v\right\|_{r}^{2}\right), & \\
& \forall v \in \mathcal{F}^{p, 1}(M) \cap\left(\mathcal{N}_{F}^{p, 1}(M)\right)^{\perp} .
\end{aligned}\right.
$$

[The orthogonal is taken with respect to the $L^{2}$-scalar product on $\mathcal{F}^{p, 1}(M)$.]

- $H^{p, 1}\left(M, F_{M}\right) \simeq \mathcal{N}_{F}^{p, 1}(M)$.

Proof. The first statement is a consequence of (5.2): this subelliptic estimate for $r=0$ implies in particular that the $L^{2}$ and $W^{1 / 2}$-Sobolev norms are equivalent on $\mathcal{N}_{\mathrm{F}}^{p, 1}(\mathrm{M})$. Then by Rellich's theorem the unit $L^{2}$ ball in $\mathcal{N}_{\mathrm{F}}^{p, 1}(\mathrm{M})$ is compact and this implies that $\mathcal{N}_{\mathrm{F}}^{p, 1}(\mathrm{M})$ is finite dimensional and thus also equals its weak closure with respect to the $L^{2}$ norm. 
The second statement can be proved by contradiction: assume that there are real numbers $r, r^{\prime}$, with $-(1 / 2) \leq r^{\prime}<r, r \geq 0$, for which $(5.5)$ is not valid for any positive constant $C_{r, r^{\prime}, p}$.

Then we can find a sequence $\left\{v_{v}\right\} \subset \mathcal{F}^{p, 1}(\mathrm{M}) \cap\left(\mathcal{N}_{\mathrm{F}}^{p, 1}(\mathrm{M})\right)^{\perp}$ with

$$
\left\|v_{v}\right\|_{r^{\prime}+(1 / 2)}=1, \quad\left\|\bar{\partial}_{\mathrm{M}}^{\mathrm{F}} v\right\|_{r}^{2}+\left\|\left(\bar{\partial}_{\mathrm{M}}^{\mathrm{F}}\right)^{*} v\right\|_{r}^{2}<2^{-v} .
$$

By (5.2) this sequence is bounded in the $\mathrm{W}^{r+(1 / 2)}$-norm and therefore has a subsequence $\left\{v_{v^{\prime}}\right\}$ which, weakly in $\mathbf{W}^{r+(1 / 2)}$ and hence strongly in $\mathbf{W}^{r^{\prime}+(1 / 2)}$, converges to a $v_{\infty}$. In particluar $\left\|v_{\infty}\right\|_{r^{\prime}+(1 / 2)}=1$ shows that $v_{\infty} \neq 0$. This limit is a weak solution of $\bar{\partial}_{\mathrm{M}}^{\mathrm{F}} v_{\infty}=0$ and $\left(\bar{\partial}_{\mathrm{M}}^{\mathrm{F}}\right)^{*} v_{\infty}=0$ and therefore, by the hypoellipticity of $\bar{\partial}_{\mathrm{M}}^{\mathrm{F}} \oplus\left(\bar{\partial}_{\mathrm{M}}^{\mathrm{F}}\right)^{*}$ following from the subellipticity estimate (5.2), is a smooth section and thus a nonzero element of $\mathcal{N}_{\mathrm{F}}^{p, 1}(\mathrm{M})$.

This completes the proof of the second statement.

It is clear that the elements of $\mathcal{N}_{\mathrm{F}}^{p, 1}(\mathrm{M})$ represent cohomology classes in $H^{p, 1}\left(\mathrm{M}, \mathrm{F}_{\mathrm{M}}\right)$. Indeed, if $v \in \mathcal{N}_{\mathrm{F}}^{p, 1}$ and $v=\bar{\partial}_{\mathrm{M}}^{\mathrm{F}} u$ for some $u \in \mathcal{F}^{p, 0}(\mathrm{M})$, then

$$
\left(\bar{\partial}_{\mathrm{M}}^{\mathrm{F}}\right)^{*} \bar{\partial}_{\mathrm{M}}^{\mathrm{F}} u=0 \Longrightarrow\|v\|_{0}^{2}=\left(v \mid \bar{\partial}_{\mathrm{M}}^{\mathrm{F}} u\right)_{0}=\left(\left(\bar{\partial}_{\mathrm{M}}^{\mathrm{F}}\right)^{*} v \mid u\right)_{0}=0
$$

shows that $v=0$. On the other hand, if $f \in \mathcal{F}^{p, 1}(\mathrm{M})$ and $\bar{\partial}_{\mathrm{M}}^{\mathrm{F}} f=0$, then we can decompose $f$ into the sum $f=f_{0}+f_{1}$, with $f_{0} \in \mathcal{N}_{\mathrm{F}}^{p, 1}(\mathrm{M})$ and $f_{1} \in\left(\mathcal{N}_{\mathrm{F}}^{p, 1}(\mathrm{M})\right)^{\perp}$.

We get

$$
\begin{aligned}
\left|\left(f_{1} \mid v\right)_{0}\right| \leq\left\|f_{1}\right\|_{0}\|v\|_{0} \leq C_{-1 / 2,0, p}\left(\left\|\bar{\partial}_{\mathrm{M}}^{\mathrm{F}} v\right\|^{2}+\left\|\left(\bar{\partial}_{\mathrm{M}}^{\mathrm{F}}\right)^{*} v\right\|^{2}\right)^{1 / 2} \\
\forall v \in \mathcal{F}^{p, 1}(\mathrm{M}) \cap\left(\mathcal{N}_{\mathrm{F}}^{p, 1}(\mathrm{M})\right)^{\perp} .
\end{aligned}
$$

By Riesz' representation theorem there is a unique $w \in \mathcal{F}^{p, 1}(\mathrm{M}) \cap\left(\mathcal{N}_{\mathrm{F}}^{p, 1}(\mathrm{M})\right)^{\perp}$ ( $w$ is smooth because of the subellipticity estimate (5.2) ) such that

$$
\left(\bar{\partial}_{\mathrm{M}}^{\mathrm{F}} w \mid \bar{\partial}_{\mathrm{M}}^{\mathrm{F}} v\right)_{0}+\left(\left(\bar{\partial}_{\mathrm{M}}^{\mathrm{F}}\right)^{*} w \mid\left(\bar{\partial}_{\mathrm{M}}^{\mathrm{F}}\right)^{*} v\right)_{0}=\left(f_{1} \mid v\right), \forall v \in \mathcal{F}^{p, 1}(\mathrm{M}) \cap\left(\mathcal{N}_{\mathrm{F}}^{p, 1}(\mathrm{M})\right)^{\perp} .
$$

Since $f_{1}$ is $L^{2}$-orthogonal to $\mathcal{N}_{\mathrm{F}}^{p, 1}(\mathrm{M})$, this equality holds true for all $v$ in $\mathcal{F}^{p, 1}(\mathrm{M})$ and integration by parts yields

$$
\left(\bar{\partial}_{\mathrm{M}}^{\mathrm{F}}\right)^{*} \bar{\partial}_{\mathrm{M}}^{\mathrm{F}} w=0, \quad \bar{\partial}_{\mathrm{M}}^{\mathrm{F}}\left(\bar{\partial}_{\mathrm{M}}^{\mathrm{F}}\right)^{*} w=f_{1} .
$$

This shows that the orthogonal projection of $\operatorname{ker}\left(\bar{\partial}_{\mathrm{M}}^{\mathrm{F}}: \mathcal{F}^{p, 1}(\mathrm{M}) \rightarrow \mathcal{F}^{p, 2}(\mathrm{M})\right)$ onto $\mathcal{N}_{\mathrm{F}}^{p, 1}(\mathrm{M})$ yields, by passing to the injective quotient, an isomorphism between $H^{p, 1}(\mathrm{M}, \mathrm{F})$ and $\mathcal{N}_{\mathrm{F}}^{p, 1}(\mathrm{M})$.

The proof is complete.

Likewise, we have a similar statement for $C R$ sections of $\mathrm{F}$ on $\mathrm{M}$. Set

$$
\mathcal{N}_{\mathrm{F}}^{p, 0}(\mathrm{M})=\left\{u \in \mathcal{F}^{p, 0}(\mathrm{M}) \mid \bar{\partial}_{\mathrm{M}}^{\mathrm{F}} u=0\right\} .
$$

Proposition 5.4. Under the assumptions of Proposition 5.2.

- The space $\mathcal{N}_{F}^{p, 0}(M)$ is finite dimensional and equals its weak closure with respect to the $L^{2}$ norm; 
- For every pair of real numbers $r, r^{\prime}$ with $-(1 / 2) \leq r^{\prime}<r, r \geq 0$, there is a constant $C_{r, r^{\prime}, p}>0$ such that

$$
\|u\|_{r^{\prime}+(1 / 2)} \leq C_{r, r^{\prime} p}\left\|\bar{\partial}_{M}^{F} u\right\|_{r}^{2}, \quad \forall u \in \mathcal{F}^{p, 0}(M) \cap\left(\mathcal{N}_{F}^{p, 0}(M)\right)^{\perp} .
$$

5.3. Deformation of tangential $C R$ complexes. Let us consider the situation described at the beginning of $\$ 5.2$. Here we make the additional assumption that $\mathrm{M}$ is a generic compact smooth $C R$ submanifold of a complex variety $\mathrm{X}$ of dimension $n+k$ and that the complex $C R$ line bundle $\mathrm{F}_{\mathrm{M}}$ is the pullback of a holomorphic line bundle $F_{X}$ on $X$, associated to a global meromorphic function on $\mathrm{X}$, whose pole divisor has support $D$. We indicate by $\mathrm{M}_{0}$ the $C R$ structure, of type $(n, k)$, induced on $\mathrm{M}$ by the embedding $\mathrm{M} \hookrightarrow \mathrm{X}$ and consider a smooth one-parameter deformation $\left\{\mathrm{M}_{\mathrm{t}}\right\}$ on $\mathrm{M}$ of this $C R$ structure $\mathrm{M}_{0}$, with the constraint:

The $C R$ structures of $\mathrm{M}_{\mathrm{t}}$ agree to infinite order on $\mathrm{M} \cap D$.

We observed in $\$ 3.3$ that in this case $F_{M}$ can be also considered as a $C R$ line bundle on each $\mathrm{M}_{\mathrm{t}}$. We need to consider the different tangential Cauchy-Riemann complexes with coefficients in $\mathrm{F}_{s f M}$ as complexes of partial differential operators acting on the same spaces of vector valued functions.

We showed in $\$ 3.1$ that we can represent the tangential $C R$ complex (on the trivial line bundle) by fixing a complement $\mathrm{E}_{\mathrm{M}}=\mathrm{E}_{\mathrm{M}}^{0,1}$ of $\mathrm{T}_{\mathrm{M}}^{1,0}$. This yields isomorphisms between $\mathrm{E}_{\mathrm{M}}^{p, q}=\Lambda^{q}\left(\mathrm{E}_{\mathrm{M}}\right) \wedge_{\mathrm{M}} \Lambda^{p}\left(\mathrm{~T}_{\mathrm{M}}^{1,0}\right)$ and $\mathrm{Q}_{\mathrm{M}}^{p, q}$ for all integers $p, q$ with $0 \leq p \leq n+k, 0 \leq q \leq n$.

We will be interested in deformations $\mathrm{M}_{\mathrm{t}}$ corresponding to small values of the parameter $t$. In particular, after shrinking, we can assume that $E_{M}$ is transversal to $\mathrm{T}_{\mathrm{M}_{\mathrm{t}}}^{1,0}$ for all values of the parameter $\mathrm{t}$. Then the tangential $C R$ complexes

$$
0 \longrightarrow \mathcal{E}_{\mathrm{M}}^{0,0} \stackrel{\mathrm{d}_{\mathrm{M}_{\mathrm{t}}}^{\prime \prime}}{\longrightarrow} \mathcal{E}_{\mathrm{M}}^{0,1} \stackrel{\mathrm{d}_{\mathrm{M}_{\mathrm{t}}}^{\prime \prime}}{\longrightarrow} \cdots \rightarrow \mathcal{E}_{\mathrm{M}}^{0, n-1} \stackrel{\mathrm{d}_{\mathrm{M}_{\mathrm{t}}}^{\prime \prime}}{\longrightarrow} \mathcal{E}_{\mathrm{M}}^{0, n} \longrightarrow 0
$$

are defined as complexes of linear partial differential operators, smoothly depending on the parameter $t$, which act on the germs of sections of the same fiber bundles.

To obtain an analogous presentation for positive values of $p$, we note that every $\tau$ in $\mathrm{T}_{\mathrm{M}_{\mathrm{t}}}^{1,0}$ uniquely decomposes into the sum $\tau^{\prime}+\tau^{\prime \prime}$ of a $\tau^{\prime} \in \mathrm{T}_{\mathrm{M}_{0}}^{1,0}$ and $\tau^{\prime \prime} \in \mathrm{E}_{\mathrm{M}}$. The correspondence $\tau \leftrightarrow \tau^{\prime}$ yields smooth isomorphisms $\mathrm{Q}_{\mathrm{M}_{\mathrm{t}}, 0}^{p, 0} \simeq \mathrm{Q}_{\mathrm{M}_{0}}^{p, 0}$ of complex vector bundles, from which we obtain also for positive $p$ representations

$$
0 \longrightarrow \mathcal{E}_{\mathrm{M}}^{p, 0} \stackrel{\mathrm{d}_{\mathrm{M}_{\mathrm{t}}}^{\prime \prime}}{\longrightarrow} \mathcal{E}_{\mathrm{M}}^{p, 1} \stackrel{\mathrm{d}_{\mathrm{M}_{\mathrm{t}}}^{\prime \prime}}{\longrightarrow} \cdots \rightarrow \mathcal{E}_{\mathrm{M}}^{0, n-1} \stackrel{\mathrm{d}_{\mathrm{M}_{\mathrm{t}}}^{\prime \prime}}{\longrightarrow} \mathcal{E}_{\mathrm{M}}^{p, n} \longrightarrow 0
$$

of the tangential $C R$ complexes on $\mathrm{M}_{\mathrm{t}}$ by linear partial differential operators acting on the same complex vector bundles and smoothly depending on the parameter $\mathrm{t}$. 
Finally, for the line bundle $F_{M}$, we use the trivialization given by the sections $\sigma_{i}$ of $\$ 3.3$ and (3.3) to compute their $\bar{\partial}_{\mathrm{M}_{\mathrm{t}}}^{\mathrm{F}}$. The form $\phi_{i}^{(\mathrm{t})} \in Q^{0,1}\left(U_{i}\right)$ decomposes into the sum of a $\psi_{i}^{(\mathrm{t})} \in \mathcal{E}^{0,1}\left(U_{i}\right)$ and a form in $\Gamma^{\infty}\left(U_{i}, \mathrm{~T}_{\mathrm{M}_{\mathrm{t}}}^{1,0}\right)$.

Then we can define for every $0 \leq p \leq n+k$ and $0 \leq q<n$ and all values of the parameter $t$ a linear partial differential operators $d_{M_{t}}^{\prime \prime F}$ mapping germs of smooth sections of $F_{M} \otimes E_{M}^{p, q}$ to germs of smooth sections of $F_{M} \otimes E_{M}^{p, q+1}$ in such a way that

$$
\mathrm{d}_{\mathrm{M}_{\mathrm{t}}}^{\prime \prime \mathrm{F}}\left(\alpha \cdot \sigma_{i}\right)=\left(\mathrm{d}_{\mathrm{M}_{\mathrm{t}}}^{\prime \prime} \alpha+(-1)^{p+q} \psi_{i}^{(\mathrm{t})} \wedge \alpha\right) \cdot \sigma_{i}, \quad \forall i, \forall \alpha \in \mathcal{E}_{\mathrm{M}}^{p, q}\left(U_{i}\right) .
$$

In this way we represent the different tangential $C R$ complexes for forms with coefficients in $F_{M}$ as complexes of differential operators, smoothly depending on the parameter $t$, but acting on the same smooth complex vector bundles:

$$
\begin{aligned}
0 \rightarrow \mathcal{E}^{p, 0}\left(\mathrm{M}, \mathrm{F}_{\mathrm{M}}\right) & \stackrel{\mathrm{d}_{\mathrm{M}_{\mathrm{t}}}^{\prime \prime \mathrm{F}}}{\longrightarrow} \mathcal{E}^{p, 1}\left(\mathrm{M}, \mathrm{F}_{\mathrm{M}}\right) \rightarrow \cdots \\
\cdots & \rightarrow \mathcal{E}^{p, n-1}\left(\mathrm{M}, \mathrm{F}_{\mathrm{M}}\right) \stackrel{\mathrm{d}_{\mathrm{M}_{\mathrm{t}}}^{\prime \prime \mathrm{F}}}{\longrightarrow} \mathcal{E}^{p, n}\left(\mathrm{M}, \mathrm{F}_{\mathrm{M}}\right) \rightarrow 0 .
\end{aligned}
$$

5.4. Estimates for deformations. We keep the notation and assumptions of $\$ 5.3$. Our goal is to show that the results of Propositions 5.25.3 remain valid for small deformations.

Proposition 5.5. Let $M$ be a compact generic $C R$ submanifold of type $(n, k)$ of a complex variety $X$. Let $F_{X}$ be the holomorphic line bundle associated to a global meromorphic function on $X$ and $D$ the support of its polar divisor. We assume that $M$ is 2-pseudoconcave. Let $\left\{M_{t}\right\}$ be a deformation of the $C R$ structure induced on $M=M_{0}$ by the embedding into $X$, smoothly depending on the parameter $t$ and with $C R$ structures agreeing to infinite order on $D \cap M$. Then we can find $\epsilon_{0}>0$ such that for all $|t|<\epsilon_{0}$

(1) for every $0 \leq p \leq n+k$ and every real $r \geq 0$ there is a positive constant $c_{r, p}>0$ such that

$$
\left\{\begin{array}{l}
c_{r, p} \cdot\|u\|_{r+(1 / 2)}^{2} \leq\left\|d_{M_{t}}^{\prime \prime F} u\right\|_{r}^{2}+\|u\|_{0}^{2}, \quad \forall u \in \mathcal{F}^{p, 0}(M) \\
c_{r, p} \cdot\|v\|_{r+(1 / 2)}^{2} \leq\left\|d_{M_{t}}^{\prime \prime F}\right\|_{r}^{2}+\left\|\left(d_{M_{t}}^{\prime \prime F}\right)^{*} v\right\|_{r}^{2}+\|v\|_{0}^{2}, \quad \forall v \in \mathcal{F}^{p, 1}(M) .
\end{array}\right.
$$

(2) for every pair $r^{\prime}, r$ of real numbers with $-(1 / 2) \leq r^{\prime}<r, r \geq 0$ and $0 \leq p \leq n+k$, there is a positive constant $C_{r, r, p}>0$ such that

$$
\left\{\begin{aligned}
&\|u\|_{r^{\prime}+(1 / 2)}^{2} \leq C_{r, r^{\prime}, p}\left\|d_{M_{t}}^{\prime F} u\right\|_{r}^{2} \forall u \in \mathcal{F}^{p, 0}(M) \cap\left(\mathcal{N}_{F}^{p, 0}\left(M_{t}\right)\right)^{\perp} \\
&\|v\|_{r^{\prime}+(1 / 2)}^{2} \leq C_{r, r^{\prime}, p}\left(\left\|d_{M_{t}}^{\prime F} v\right\|_{r}^{2}+\left\|\left(d_{M_{t}}^{\prime F}\right)^{*} v\right\|_{r}^{2}\right) \\
& \forall v \in \mathcal{F}^{p, 1}(M) \cap\left(\mathcal{N}_{F}^{p, 1}\left(M_{t}\right)\right)^{\perp} .
\end{aligned}\right.
$$

(3) $\operatorname{dim}_{\mathbb{C}}\left(H^{p, 0}\left(M_{t}, F_{M}\right)\right) \leq \operatorname{dim}_{\mathbb{C}}\left(H^{p, 0}\left(M_{0}, F_{M}\right)\right)<+\infty$ and $\operatorname{dim}_{\mathbb{C}}\left(H^{p, 1}\left(M_{t}, F_{M}\right)\right) \leq \operatorname{dim}_{\mathbb{C}}\left(H^{p, 1}\left(M_{0}, F_{M}\right)\right)<+\infty$ for all $0 \leq p \leq n+k$. 
Proof. The proof of the subelliptic estimates in [21] yield (5.8), the constants being uniform for $|t|<\epsilon_{0}$ because they depend upon the coefficients of the linear differential operators $\mathrm{d}_{\mathrm{M}_{\mathrm{t}}}^{\prime \prime \mathrm{F}}$ and their derivatives.

We prove (2) and (3) in the case of forms $(q=1)$, as the case of sections $(q=0)$ is analogous and even simpler.

We begin by proving that, for any pair $r, r^{\prime}$ with $(-1 / 2)<r^{\prime}<r, r \geq 0$ and for all $0 \leq p \leq n+k$, we can find $\epsilon>0$ and $C_{r, r^{\prime}, p}>0$ such that

$$
\begin{aligned}
\|v\|_{r^{\prime}+(1 / 2)}^{2} \leq & C_{r, r^{\prime}, p}\left(\left\|\mathrm{~d}_{\mathrm{M}_{\mathrm{t}}}^{\prime \prime \mathrm{F}} v\right\|_{r}^{2}+\left\|\left(\mathrm{d}_{\mathrm{M}_{\mathrm{t}}}^{\prime \prime \mathrm{F}}\right)^{*} v\right\|_{r}^{2}\right), \\
& \forall|\mathrm{t}|<\epsilon, \quad \forall v \in \mathcal{E}^{p, 1}\left(\mathrm{M}, \mathrm{F}_{\mathrm{M}}\right) \cap\left(\mathcal{N}_{\mathrm{F}}^{p, 1}\left(\mathrm{M}_{0}\right)\right)^{\perp} .
\end{aligned}
$$

We argue by contradiction: if this was false, we could find a sequence $\left\{v_{v}\right\}$ in $\mathcal{E}^{p, 1}\left(\mathrm{M}, \mathrm{F}_{\mathrm{M}}\right) \cap\left(\mathcal{N}_{\mathrm{F}}^{p, 1}\left(\mathrm{M}_{0}\right)\right)^{\perp}$ and a sequence $\left\{\mathrm{t}_{v} \rightarrow 0\right\}$ such that

$$
\left\|v_{v}\right\|_{r^{\prime}+(1 / 2)}^{2}=1, \quad\left\|\mathrm{~d}_{\mathrm{M}_{\mathrm{t}}}^{\prime \prime} v_{v}\right\|_{r}^{2}+\left\|\left(\mathrm{d}_{\mathrm{M}_{\mathrm{t}}}^{\prime \prime}\right)^{*} v_{v}\right\|_{r}^{2}<2^{-v}
$$

By (1), the sequence $\left\{v_{v}\right\}$ is uniformly bounded in $\mathbf{W}^{r+1 / 2}$ and then, by the compactness of the inclusion $\mathbf{W}^{r+(1 / 2)} \hookrightarrow \mathbf{W}^{r^{\prime}+(1 / 2)}$, contains a subsequence which strongly converges in $W^{r^{\prime}+(1 / 2)}$ to a $v_{\infty}$, which on one hand should be different from 0 because $\left\|v_{\infty}\right\|_{r^{\prime}+(1 / 2)}=1$, on the other hand should be zero because it belongs to $\mathcal{N}_{\mathrm{F}}^{p, 1}\left(\mathrm{M}_{0}\right) \cap\left(\mathcal{N}_{\mathrm{F}}^{p, 1}\left(\mathrm{M}_{0}\right)\right)^{\perp}$.

Estimate (5.10) implies that $\mathcal{N}_{\mathrm{F}}^{1, p}\left(\mathrm{M}_{\mathrm{t}}\right) \cap\left(\mathcal{N}_{\mathrm{F}}^{p, 1}\left(\mathrm{M}_{0}\right)\right)^{\perp}=\{0\}$ for $|\mathrm{t}|<\epsilon$. This yields (3), because the dimension of $H^{1, p}\left(\mathrm{M}_{\mathrm{t}}, \mathrm{F}\right)$ equals that of $\mathcal{N}_{\mathrm{F}}^{1, p}\left(\mathrm{M}_{\mathrm{t}}\right)$.

We can prove (2) by an argument similar to the one used for (5.10).

5.5. Vanishing theorems. We will use Proposition 5.5 to solve $\mathrm{d}_{M_{t}}^{\prime \prime} u_{t}=f$, for forms $f$ in $\mathcal{E}^{0,1}\left(\mathrm{M}, \mathrm{F}_{\mathrm{M}}\right)$, with $u_{\mathrm{t}}$ satisfying uniform estimates in the Sobolev norms $\mathrm{W}^{r}$, for sufficiently large $r$, yielding by the immersion theorems control upon the derivatives of $u_{\mathrm{t}}$.

Theorem 5.6. Let us keep the assumptions of Proposition 5.5 Assume moreover that $F_{M}$ is a positive line bundle on $X$. Then we can find $\epsilon_{0}>0$ such that

$$
H^{p, q}\left(M_{t}, F_{M}^{-\ell}\right)=0, \quad \forall \ell \geq \ell_{0}, 0 \leq p \leq n+k, q=0,1, \quad|t|<\epsilon_{0} .
$$

Moreover, for every $f \in \mathcal{E}^{0,1}\left(M, F_{M}^{-\ell}\right)$ satisfying $d_{M_{t}}^{\prime F} f=0$ there is a unique $u_{t}$ in $\Gamma^{\infty}\left(M, F^{-\ell}\right)$ such that, for $r^{\prime}<r$ and $r \geq 1 / 2$,

$$
d_{M_{t}}^{\prime F} u_{t}=f \quad \text { and } \quad\left\|u_{t}\right\|_{r^{\prime}} \leq C_{r^{\prime}, r}\|f\|_{r-(1 / 2)} .
$$

Proof. The first statement is a consequence of Theorem 5.1 and Proposition 5.5. These results allow us to fix $\epsilon_{0}$ in such a way that, by (5.9),

$$
\|v\|_{\mathrm{t}}=\left(\left\|\mathrm{d}_{\mathrm{M}_{\mathrm{t}}}^{\prime \prime \mathrm{F}} v\right\|_{0}^{2}+\left\|\left(\mathrm{d}_{\mathrm{M}_{\mathrm{t}}}^{\prime \prime \mathrm{F}}\right)^{*} v\right\|_{0}\right)^{1 / 2}
$$

is a pre-Hilbertian norm on $\mathrm{E}^{0,1}\left(\mathrm{M}_{\mathrm{t}}, \mathrm{F}_{\mathrm{M}}^{-\ell}\right)$. Let $\mathbf{S}_{\mathrm{t}}$ be its completion with respect to this norm. Given $f \in \mathrm{E}^{0,1}\left(\mathrm{M}, \mathrm{F}_{\mathrm{M}}^{-\ell}\right)$, we can consider $v \rightarrow(v \mid f)_{0}$ as a 
linear continuous functional on $\mathbf{S}_{\mathrm{t}}$ and hence by Riesz representation Theorem there is a unique $w \in \mathbf{S}_{\mathrm{t}}$ such that

$$
(v \mid f)_{0}=\left(\mathrm{d}_{\mathrm{M}_{\mathrm{t}}}^{\prime \prime \mathrm{F}} v \mid \mathrm{d}_{\mathrm{M}_{\mathrm{t}}}^{\prime \prime \mathrm{F}} w\right)_{0}+\left(\left(\mathrm{d}_{\mathrm{M}_{\mathrm{t}}}^{\prime \prime \mathrm{F}}\right)^{*} v \mid\left(\mathrm{d}_{\mathrm{M}_{\mathrm{t}}}^{\prime \prime \mathrm{F}}\right)^{*} w\right)_{0}, \quad \forall v \in \mathrm{E}^{0,1}\left(\mathrm{M}, \mathrm{F}_{\mathrm{M}}^{-\ell}\right) .
$$

By the subelliptic estimate (5.8), in fact $w \in \mathrm{E}^{0,1}\left(\mathrm{M}, \mathrm{F}_{\mathrm{M}}^{-\ell}\right)$, i.e. $w$ is smooth, because we assumed that $f$ is smooth. If $f$ satisfies the integrability condition $\mathrm{d}_{M_{\mathrm{t}}}^{\prime \prime \mathrm{F}} f=0$, then integration by parts yields

$$
\begin{aligned}
0 & =\left(d_{M_{t}}^{\prime \prime F} w \mid d_{M_{t}}^{\prime \prime F} f\right)_{0}=\left(\left(d_{M_{t}}^{\prime F}\right)^{*} d_{M_{t}}^{\prime \prime F} w \mid f\right) \\
& =\left(d_{M_{t}}^{\prime \prime F}\left(d_{M_{t}}^{\prime \prime F}\right)^{*} d_{M_{t}}^{\prime \prime F} w \mid d_{M_{t}}^{\prime \prime F} w\right)_{0}+\left(\left(d_{M_{t}}^{\prime \prime F}\right)^{*}\left(d_{M_{t}}^{\prime \prime F}\right)^{*} d_{M_{t}}^{\prime \prime F} w \mid\left(d_{M_{t}}^{\prime \prime F}\right)^{*} w\right)_{0} \\
& =\left(\left(d_{M_{t}}^{\prime \prime F}\right)^{*} d_{M_{t}}^{\prime \prime F} w \mid\left(d_{M_{t}}^{\prime \prime F}\right)^{*} d_{M_{t}}^{\prime \prime F} w\right)_{0} \Longrightarrow\left(d_{M_{t}}^{\prime F}\right)^{*} d_{M_{t}}^{\prime \prime F} w=0 \\
& \Longrightarrow 0=\left(\left(d_{M_{t}}^{\prime \prime F}\right)^{*} d_{M_{t}}^{\prime \prime F} w \mid w\right)_{0}=\left\|d_{M_{t}}^{\prime F} w\right\|_{0}^{2} \Longrightarrow d_{M_{t}}^{\prime \prime F} w=0 .
\end{aligned}
$$

By (5.9), for all $0<r<1 / 2$

$$
\begin{aligned}
\left(u_{\mathrm{t}} \mid u_{\mathrm{t}}\right)_{0} & =\left(\left(\mathrm{d}_{\mathrm{M}_{\mathrm{t}}}^{\prime \prime \mathrm{F}}\right)^{*} w,\left(\mathrm{~d}_{M_{\mathrm{t}}}^{\prime \prime \mathrm{F}}\right)^{*} w\right)_{0}=\left(w, \mathrm{~d}_{\mathrm{M}_{\mathrm{t}}}^{\prime \prime \mathrm{F}}\left(\mathrm{d}_{M_{\mathrm{t}}}^{\prime \prime \mathrm{F}}\right)^{*} w\right)=(w, f)_{0} \\
& \leq\|f\|_{-r}\|w\|_{r} \leq C_{r}^{\prime \prime}\|f\|_{-r}\left\|u_{\mathrm{t}}\right\|_{r-\frac{1}{2}} \leq C_{r}^{\prime \prime}\|f\|_{-r}\left\|u_{\mathrm{t}}\right\|_{0}
\end{aligned}
$$

Hence

$u_{\mathrm{t}}=\left(\mathrm{d}_{\mathrm{M}_{\mathrm{t}}}^{\prime \prime \mathrm{F}}\right)^{*} w \quad$ solves $\quad \mathrm{d}_{\mathrm{M}_{\mathrm{t}}}^{\prime \prime \mathrm{F}} u_{\mathrm{t}}=f \quad$ and $\quad\left\|u_{\mathrm{t}}\right\|_{0} \leq C_{r}^{\prime \prime}\|f\|_{-r}, \quad \forall 0<r<1 / 2$, with constants $C_{r}^{\prime \prime}$ independent of $|\mathrm{t}|<\epsilon_{0}$. Since $\mathscr{O}\left(\mathrm{M}_{\mathrm{t}}, \mathrm{F}_{\mathrm{M}}\right)=\{0\}$, the solution is unique and therefore we obtain the estimates in (5.12) by recurrence, using, for any pseudodifferential operator $P$ on $\mathrm{M}$, the identity

$$
\mathrm{d}_{\mathrm{M}_{\mathrm{t}}}^{\prime \prime \mathrm{F}} P\left(u_{\mathrm{t}}\right)=\left[\mathrm{d}_{\mathrm{M}_{\mathrm{t}}}^{\prime \prime \mathrm{F}}, P\right]\left(u_{\mathrm{t}}\right)+P(f) \text {. }
$$

If $P$ has order $r^{\prime}$, with $0<r^{\prime}<1 / 2$, then

$$
\left\|P\left(u_{\mathrm{t}}\right)\right\|_{0} \leq C_{r^{\prime}}^{\prime \prime}\left(\left\|\left[\mathrm{d}_{\mathrm{M}_{\mathrm{t}}}^{\prime \prime \mathrm{F}}, P\right]\left(u_{\mathrm{t}}\right)\right\|_{-r^{\prime}}+\|P(f)\|_{-r^{\prime}}\right) \leq \operatorname{const}_{P} C_{r^{\prime}}^{\prime \prime}\left(\left\|u_{\mathrm{t}}\right\|_{0}+\|f\|_{0}\right),
$$

with a constant const $_{P}$ which only depends on $P$. This yields

$$
\left\|u_{\mathrm{t}}\right\|_{r^{\prime}} \leq C_{r^{\prime},(1 / 2)}\|f\|_{0} \text {. }
$$

with constants independent of $t$. Repeating this argument we obtain the estimate in the statement.

\section{Proof of Theorem 1.1}

6.1. The general case. We keep the notation of $\$ 3.3$, The $\left(f_{i}^{\prime \prime}\right)$ define a global section $\sigma$ of $\mathrm{F}_{\mathrm{M}}$, which is $C R$ for the structure on $\mathrm{M}_{0}$ and is $\neq 0$ on $\mathrm{M} \backslash D$.

Fix an integer $\ell$ such that $\mathrm{F}_{\mathrm{X}}^{\ell}$ is very ample and $H^{0,1}\left(\mathrm{M}_{\mathrm{t}}, \mathrm{F}_{\mathrm{M}}^{-h}\right)=0$ for $h \geq \ell$ and $|t|<\epsilon_{0}$, which is possible by Theorem 5.6.

For every $t$ we set

$$
g_{\mathrm{t}}= \begin{cases}\mathrm{d}_{\mathrm{M}_{\mathrm{t}}^{\prime}}^{\prime \mathrm{F}^{-\ell}} \sigma^{-\ell}, & \text { on } \mathrm{M} \backslash D, \\ 0, & \text { on } D .\end{cases}
$$

Since the $C R$ structure of $\mathrm{M}_{\mathrm{t}}$ agrees with that of $\mathrm{M}_{0}$ to infinite order on $D$, the $g_{\mathrm{t}}$ 's are smooth sections in $\mathcal{E}^{0,1}\left(\mathrm{M}, \mathrm{F}^{-\ell}\right)$ that vanish to infinite order on 
$D$ and satisfy the integrability condition $\mathrm{d}_{\mathrm{M}_{\mathrm{t}}^{\prime}}^{\prime \mathrm{F}^{-\ell}} g_{\mathrm{t}}=0$. By Theorem 5.6, for $|\mathrm{t}|<\epsilon_{0}$, we can find $v_{\mathrm{t}} \in \Gamma^{\infty}\left(\mathrm{M}, \mathrm{F}_{\mathrm{M}}^{-\ell}\right)$ to solve

$$
\mathrm{d}_{\mathrm{M}_{\mathrm{t}}^{\prime}}^{\prime \mathrm{F}^{-\ell}} v_{i, \mathrm{t}}=g_{\mathrm{t}} \text {, and we have }\left\|v_{\tau}\right\|_{r^{\prime}} \leq C_{r^{\prime}, r}\left\|g_{\mathrm{t}}\right\|_{r-(1 / 2)}
$$

for $0 \leq r^{\prime}<r$, with a constant $C_{r, r^{\prime}}$ independent of $\mathrm{t}$. Then

$$
\sigma^{-\ell}-v_{\mathrm{t}} \in \mathscr{O}_{\mathrm{M}_{\mathrm{t}}}\left(\mathrm{M}_{\mathrm{t}} \backslash D, \mathrm{~F}_{\mathrm{M}}^{-\ell}\right) \text {. }
$$

By Sobolev's embedding theorems, the sup-norms of the first derivatives of the $v_{i, \mathrm{t}}$ are bounded by their $\mathrm{W}^{r^{\prime}}$-norms for $r^{\prime}>(2 n+k+2) / 2$. As $\left\|g_{i, \mathrm{t}}\right\|_{r-(1 / 2)} \rightarrow 0$ for $\mathrm{t} \rightarrow 0$, we obtain that, for $|\mathrm{t}| \ll 1$, the sections

$$
\eta_{\mathrm{t}}=\left(\sigma^{-\ell}-v_{\mathrm{t}}\right)^{-1} \in \mathscr{O}_{\mathrm{M}_{\mathrm{t}}}\left(\mathrm{M}_{\mathrm{t}} \backslash D, \mathrm{~F}_{\mathrm{M}}^{\ell}\right) \text {. }
$$

extend to smooth sections of $\mathscr{O}_{\mathrm{M}_{\mathrm{t}}}\left(\mathrm{M}_{\mathrm{t}}, \mathrm{F}_{\mathrm{M}}^{\ell}\right)$, defining the divisor $D$.

Since $\mathrm{F}_{\mathrm{X}}^{\ell}$ is very ample, there are sections $\sigma_{0}, \sigma_{1}, \ldots, \sigma_{m} \in \mathscr{O}_{\mathrm{M}_{0}}\left(\mathrm{X}, \mathrm{F}_{\mathrm{X}}^{\ell}\right)$ providing a holomorphic embedding of $\mathrm{X}$ into $\mathbb{C P}^{m}$.

Their restrictions $\left(\sigma_{0.0}, \sigma_{1,0}, \ldots, \sigma_{m, 0}\right)$ to $\mathrm{M}$ provide a $C R$ embedding of $\mathrm{M}_{0}$ into $\mathbb{C P}^{m}$. For every $\mathrm{t}$ and $1 \leq i \leq m$ set

$$
f_{i, \mathrm{t}}= \begin{cases}\mathrm{d}_{\mathrm{M}_{\mathrm{t}}^{\prime}}^{\prime \mathrm{F}^{-\ell}}\left(\eta_{\mathrm{t}}^{-2} \sigma_{i}\right), & \text { on } \mathrm{M} \backslash D, \\ 0, & \text { on } D .\end{cases}
$$

Then, arguing as above and invoking Theorem 5.6 for $|\mathrm{t}|<\epsilon_{0}$, we can find $u_{i, \mathrm{t}} \in \Gamma^{\infty}\left(\mathrm{M}, \mathrm{F}_{\mathrm{M}}^{-\ell}\right)$ to solve

$$
\mathrm{d}_{\mathrm{M}_{\mathrm{t}}^{\prime}}^{\prime \mathrm{F}^{-\ell}} u_{i, \mathrm{t}}=f_{i, \mathrm{t}} \text {, and we have }\left\|u_{i, \tau}\right\|_{r^{\prime}} \leq C_{r^{\prime}, r}\left\|f_{i, \mathrm{t}}\right\|_{r-(1 / 2)}
$$

for $0 \leq r^{\prime}<r$, with a constant $C_{r, r^{\prime}}$ independent of t. Then

$$
\sigma_{i, \mathrm{t}}=\sigma_{i}-u_{i, \mathrm{t}} \eta_{\mathrm{t}}^{2} \in \mathscr{O}_{\mathrm{M}_{\mathrm{t}}}\left(\mathrm{M}_{\mathrm{t}}, \mathrm{F}_{\mathrm{M}}^{\ell}\right) \text {. }
$$

As $\left\|f_{i, \mathrm{t}}\right\|_{r-(1 / 2)} \rightarrow 0$ for $\mathrm{t} \rightarrow 0$, we obtain that, for $|\mathrm{t}| \ll 1$, the sections $\left(\sigma_{0, \mathrm{t}}, \sigma_{1, \mathrm{t}}, \ldots, \sigma_{m, \mathrm{t}}\right)$, being a small $C^{1}$-perturbation of $\left(\sigma_{0,0}, \sigma_{1,0}, \ldots, \sigma_{m, 0}\right)$, still provide a $C R$ immersion of $\mathrm{M}_{\mathrm{t}}$ into $\mathbb{C P}^{m}$.

The last part of the statement follows from [23], where it is shown that the maximum degree of transcendence of the field of $C R$ meromorphic functions on $\mathrm{M}_{\mathrm{t}}$ is $n+k$ : this implies that all pseudoconcave $C R$ manifold of type $(n, k)$ having a projective embedding, can be embedded into a projective complex variety of dimension $n+k$.

6.2. Generic $C R$ submanifolds of the projective space. The line bundles on the projective space $\mathbb{C P}^{n+k}$ are parametrized (modulo equivalence) by the integers. They are all holomorphically equivalent to integral powers of the line bundle $\Theta_{\mathbb{C P}^{n+k}}$, which can be described, in the covering $\left\{U_{i}=\left\{z_{i} \neq 0\right\}\right\}$ by the transition functions $\left\{g_{i, j}=z_{j}^{-1} z_{i}\right\}$. The support of the pole divisor of a meromorphic function $f$ on $\mathbb{C P}^{n+k}$ is the set of common zeros of a homogeneous polynomial

$$
D=\left\{\wp\left(z_{0}, z_{1}, \ldots, z_{n+k}\right)=0\right\}
$$


If $\wp(z)$ has degree $d$, this is the zero locus of a section of $\Theta_{\mathbb{C P}^{n+k}}^{d}$. Replacing $\wp$ by one of its powers if necessary, we can as well assume that $d>n+k$. Then we have

$$
H^{q}\left(\mathbb{C P}^{n+k}, \Theta_{\mathbb{C P}^{n+k}}^{d}\right)=\left\{\begin{array}{l}
\left\{\psi \in \mathbb{C}\left[z_{0}, z_{1}, \ldots, z_{n+k}\right] \mid \psi(\lambda z)=\lambda^{d} \psi(z)\right\}, \\
\{0\} \text { for } q>0 .
\end{array}\right.
$$

The fact that the pullback $\Theta_{\mathrm{M}}^{d}$ of $\Theta_{\mathrm{Cp}^{n+k}}^{d}$ is a $C R$ line bundle on $\mathrm{M}_{\mathrm{t}}$ implies that also all pullbacks $\Theta_{\mathrm{M}}^{p}$ of $\Theta_{\mathbb{C P}^{n+k}}^{p}$ to $M$, for any $p \in Z$, are $C R$ line bundles on $\mathrm{M}_{\mathrm{t}}$. Indeed the fact that $\Theta_{\mathrm{M}}^{d}$ is a $C R$ line bundle on $\mathrm{M}_{\mathrm{t}}$ means that we can find an open covering of $\mathrm{M}$, that we can take of the form $\left\{U_{i, \mu}\right\}$ with $U_{i, \mu} \subseteq U_{i}=\left\{z_{i} \neq 0\right\}$ and $\mathrm{d}_{\mathrm{M}_{\mathrm{t}}}^{\prime \prime}$-closed forms $\phi_{i, \mu} \in \mathcal{E}^{0,1}\left(U_{i, \mu}\right)$ such that

$$
\left(\frac{z_{j}}{z_{i}}\right)^{d} \mathrm{~d}_{\mathrm{M}_{\mathrm{t}}}^{\prime \prime}\left(\frac{z_{i}}{z_{j}}\right)^{d}=\phi_{i, \mu}-\phi_{j, v} \quad \text { on } U_{i, \mu} \cap U_{j, v} .
$$

This is equivalent to

$$
\left(\frac{z_{j}}{z_{i}}\right) \mathrm{d}_{\mathrm{M}_{\mathrm{t}}}^{\prime \prime}\left(\frac{z_{i}}{z_{j}}\right)=\frac{1}{d} \phi_{i, \mu}-\frac{1}{d} \phi_{j, v} \quad \text { on } U_{i, \mu} \cap U_{j, v},
$$

showing that $\Theta_{\mathrm{M}}$, and therefore all $\Theta_{\mathrm{M}}^{p}$ with $p \in \mathbb{Z}$ are $C R$ line bundles on $\mathrm{M}_{\mathrm{t}}$.

By repeating the proof in $\$ 6.1$ by using the sections $\sigma_{i}=z_{i}$ of $\Theta_{\mathrm{M}}$, we obtain

Theorem 6.1. Let $M$ be a smooth compact generic $C R$ submanifold of type $(n, k)$ of $\mathbb{C} \mathbb{P}^{n+k}$ and $D$ the zero locus in $\mathbb{C P}^{n+k}$ of a homogeneous polynomial of degree d in $\mathbb{C}\left[z_{0}, z_{1}, \ldots, z_{n+k}\right]$. Let $\left\{M_{t}\right\}$ be a family of $C R$ structures of type $(n, k)$ on $M$, smoothly depending on a real parameter $t$. If

(1) $M_{0}$ is induced by the embedding $M \hookrightarrow \mathbb{C P}^{n+k}$;

(2) $M_{0}$ is 2-pseudoconcave;

(3) the CR structures of all $M_{t}$ agree to infinite order on $M \cap D$.

Then we can find $\epsilon_{0}>0$ such that, for every $|t|<\epsilon_{0}, M_{t}$ admits a generic $C R$ embedding into $\mathbb{C P}^{n+k}$.

6.3. Generic $C R$ submanifolds of Fano varieties. We recall that a complex variety $X$ is Fano if its anticanonical bundle $\mathrm{K}_{X}^{-1}$ is ample. If we add to the assumptions of Theorem 1.1 the requirement that $X$ is Fano, then we can define a projective embedding of $\mathrm{M}_{0}$ by using $C R$ sections $\sigma_{0}, \sigma_{1}, \ldots, \sigma_{m}$ of a power $\mathrm{K}_{\mathrm{M}}^{-\ell}$ of the pullback on $\mathrm{M}$ of the anticanonical bundle. Indeed, by [29], the tensor product $\mathrm{K}_{\mathrm{X}}^{-\ell} \otimes \mathrm{F}_{\mathrm{X}}^{-k}$ is the dual of a positive bundle and we can apply the arguments in $\$ 6.1$ to obtain sections $\sigma_{i, \mathrm{t}}$ in $\mathscr{O}_{\mathrm{M}_{\mathrm{t}}}\left(\mathrm{M}_{\mathrm{t}}, \mathrm{K}_{\mathrm{M}}^{-\ell}\right)$, which, for $|\mathrm{t}| \ll 1$ yield projective $C R$ embeddings $\sigma_{\mathrm{t}}=\left(\sigma_{0, \mathrm{t}}, \sigma_{1, \mathrm{t}}, \ldots, \sigma_{m, \mathrm{t}}\right)$ of $\mathrm{M}_{\mathrm{t}}$ into $\mathbb{C P}^{m}$. The images $\mathrm{M}_{\mathrm{t}}^{\prime}=\sigma_{\mathrm{t}}\left(\mathrm{M}_{\mathrm{t}}\right)$ are generic submanifolds of complex $(n+k)$-dimensional subvarieties $\mathrm{X}_{\mathrm{t}}$ of $\mathbb{C P}^{m}$ which have, by construction, an ample anticanonical bundle. Indeed we know from [23, Thm.5.2] that, since the $\mathrm{M}_{\mathrm{t}}$ are pseudoconcave, global $C R$ meromorphic functions on $\mathrm{M}_{\mathrm{t}}$ are restrictions of global meromorphic functions on $X_{t}$. Thus we have 
Theorem 6.2. Add to the assumptions of Theorem 1.1 the fact that $X$ is Fano. Then there is $\epsilon_{0}>0$ such that for all $|t|<\epsilon_{0}$ the abstract $C R$ manifold $M_{t}$ has a generic CR embedding into a complex Fano variety $X_{t}$.

Acknowledgements. The first author was supported by Deutsche Forschungsgemeinschaft (DFG, German Research Foundation, grant BR 3363/2-2).

\section{REFERENCES}

1. A. Andreotti, C.D. Hill: Complex characteristic coordinates and tangential CauchyRiemann equations. Ann. Scuola Norm. Sup. Pisa 26, 299-324 (1972)

2. T. Akahori: Deformation Theory of CR-Structures and Its Application to Deformations of Isolated Singularities I, Advanced Studies in Pure Mathematics 25, CRGeometry and Overdetermined Systems, pp. 1-40 (1997).

3. A. Altomani, C. Medori, and M. Nacinovich:, The CR structure of minimal orbits in complex flag manifolds, J. Lie Theory 16, no. 3, 483-530, (2006).

4. A.Andreotti and G.A.Fredricks: Embeddability of real analytic Cauchy-Riemann manifolds, Ann. Scuola Norm. Sup. Pisa (4) 6, 285-304, (1979).

5. A. Andreotti and H. Grauert: Theorèmes de Finitude pour la cohomologie des espaces complexes, Bulletin de la Societ'e Mathématique de France, 90, pp. 193-259 (1962).

6. H. AzAd, R. KobayAshi, M.N. QuReshi: Quasi-potentials and KhlerEinstein metrics on flag manifolds, J. Algebra 196, 620-629 (1997).

7. Au-Yeung, Yik-Hor: On Matrices Whose Nontrivial Real Linear Combinations Are Nonsingular. Proc. of the A.M.S. 29, no. 1, 17-22 (1971).

8. A. Borel, F. Hirzebruch: Characteristic classes and homogeneous spaces, Amer. J. Math 80, 459-538 (1958).

9. J. Brinkschulte, C.D. Hill: Non locally trivializable CR line bundles over compact Lorentzian CR manifolds Ann. Inst. Fourier 68, 101-108 (2018).

10. J. Brinkschulte, C.D. Hill: Inflexible CR submanifolds, Math. Z. 287, 461-472 (2017).

11. J. Brinkschulte, C.D. Hill: Flexible and inflexible CR submanifolds, Ark. Mat. 57, 23-33 (2019).

12. J. Brinkschulte, C.D. Hitl, M. Nacinovich: Obstructions to generic embeddings. Ann. Inst. Fourier 52, 1785-1792 (2002).

13. J. Brinkschulte, C.D. Hill, J. Leiterer, M. Nacinovich: Aspects of the Levi form. to appear in B.U.M.I.

14. É. CARTAN: Les systèmes différentiels exterieurs et leurs applications géométriques, Actualités Scientifiques et Industriels, ${ }^{o}$ 994, Hermann, Paris, 1945.

15. D. Catlin, L. Lempert: A note on the instability of embeddings of Cauchy-Riemann manifolds. J. Geom. Analysis 2, pp. 99-104 (1992).

16. G. Darboux: Sur le problème de Pfaff Bull. Sci. Math. 6, pp. 14-36, 49-68 (1882).

17. P. De Bartolomeis and F. Meylan: Intrinsic deformation theory of $C R$ structures. Ann. Scuola Norm. Sup. Pisa Cl. Sci. (5) IX, pp.459-494 (2010).

18. G.B. Folland, J.J. Kohn: The Neumann problem $f$ or the Cauchy-Riemann complex. Ann. Math. Studies 75, Princeton University Press, Princeton, N. J. (1972).

19. Рh. A. Griffiths, J. Harris: Principles of Algebraic Geometry, John Wiley \& Sons, New York, 1978.

20. E. HeвEY: Sobolev spaces on Riemannian manifolds, Lecture Notes in Mathematics, v.1635, Springer-Verlag, Berlin, (1996). 
21. C.D. Hill, M. Nacinovich: Pseudoconcave CR manifolds. Complex analysis and geometry (V. Ancona, E. Ballico, A. Silva, eds), Marcel Dekker, Inc., New York, 275297 (1996).

22. C.D. HiLl, M. NAcinovich: A weak pseudoconcavity condition for abstract almost $C R$ manifolds. Invent. math. 142, 251-283 (2000).

23. C.D. Hitl, M. Nacinovich: Fields of CR meromorphic functions. Rend. Sem. Mat. Univ. Padova 111, 179-204 (2004).

24. C.D. Hill, M. Nacinovich: Non completely solvable systems of complex first order PDE's. Rend. Sem. Mat. Univ. Padova 129, 129-169 (2013).

25. C.D. HiLl, G. TAIANI: Real analytic approximation of locally embeddable CR manifolds. Compositio Math. 44, 113-131 (1981).

26. L. HöRMANDER: An introduction to complex analysis in several complex variables. North Holland Mathematical Library (1990).

27. D. HusemölLer: Fibre Bundles, Graduate Texts in Mathematics, Springer, New York, (1994).

28. T. KAJISA: On the existence of local frames for CR vector bundles. Hokkaida Math. J. 42, 121-130 (2013).

29. S.L.KLeIman: Towards a numerical theory of ampleness. Ann. of Math. 84, 293-344, (1966).

30. K. Kodaira, D.C. SPencer: On deformations of complex analytic structures, I-II, III. Ann. of Math. 67, 328-466 (1958); 71, 43-76 (1960).

31. L. LeMPERT: Embeddings of three dimensional Cauchy-Riemann manifolds. Math. Ann. 300, 1-15 (1994).

32. C. Medori, M. Nacinovich: Pluriharmonic functions on abstract CR manifolds. Ann. Mat. Pura e Appl. (IV) CLXX, 377-394 (1996).

33. L. Nirenberg: On a problem of Hans Lewy. Uspeki Math. Naut. 292, pp. 241-251 (1974).

34. A. Newlander and L. Nirenberg: Complex analytic coordinates in almost complex manifolds. Annals of Mathematics. 66, pp. 391-404 (1957).

35. P.L. Polyakov Versal embeddings of compact 3-pseudoconcave CR submanifolds. Math. Z. 248, 267-312 (2004).

36. H. Rossi: Attaching analytic spaces to an analytic space along a pseudoconcave boundary, Proc. Conf. Complex Manifolds (Minneapolis), 1964, Springer-Verlag, New York, pp. 242-256 (1965).

37. J. A. Wolf, The action of a real semisimple group on a complex flag manifold. I. Orbit structure and holomorphic arc components, Bull. Amer.Math. Soc. 75 (1969), 11211237.

J. Brinkschulte: Mathematisches Institut, Universität Leipzig, Augustusplatz 10/11, 04109 LEIPZIG (GERMANY)

E-mail address: brinkschulte@math. uni-leipzig.de

C.D. Hill: Department of Mathematics, Stony Brook University, Stony Brook, N.Y. 11794 (USA)

E-mail address: dhill@math.stonybrook.edu

M. Nacinovich: Dipartimento di Matematica, II Università di Roma "Tor Vergata", Via della Ricerca Scientifica, 00133 Roma (Italy)

E-mail address: nacinovi@mat. uniroma2.it 